\title{
A systematic comparison reveals substantial differences in chromosomal versus episomal encoding of enhancer activity
}

\author{
Fumitaka Inoue, ${ }^{1,7}$ Martin Kircher, ${ }^{2,7}$ Beth Martin, ${ }^{2}$ Gregory M. Cooper, ${ }^{3}$ \\ Daniela M. Witten, ${ }^{4}$ Michael T. McManus, ${ }^{5}$ Nadav Ahituv, ${ }^{1}$ and Jay Shendure ${ }^{2,6}$ \\ ${ }^{1}$ Department of Bioengineering and Therapeutic Sciences, Institute for Human Genetics, University of California San Francisco, \\ San Francisco, California 94158, USA; ${ }^{2}$ Department of Genome Sciences, University of Washington, Seattle, Washington 98195 , \\ USA; ${ }^{3}$ HudsonAlpha Institute for Biotechnology, Huntsville, Alabama 35806, USA; ${ }^{4}$ Departments of Statistics and Biostatistics, \\ University of Washington, Seattle, Washington 98195, USA; ${ }^{5}$ Department of Microbiology and Immunology, UCSF Diabetes Center, \\ Keck Center for Noncoding RNA, University of California, San Francisco, San Francisco, California 94143, USA; ${ }^{6}$ Howard Hughes \\ Medical Institute, Seattle, Washington 98195, USA
}

\begin{abstract}
Candidate enhancers can be identified on the basis of chromatin modifications, the binding of chromatin modifiers and transcription factors and cofactors, or chromatin accessibility. However, validating such candidates as bona fide enhancers requires functional characterization, typically achieved through reporter assays that test whether a sequence can increase expression of a transcriptional reporter via a minimal promoter. A longstanding concern is that reporter assays are mainly implemented on episomes, which are thought to lack physiological chromatin. However, the magnitude and determinants of differences in cis-regulation for regulatory sequences residing in episomes versus chromosomes remain almost completely unknown. To address this systematically, we developed and applied a novel lentivirus-based massively parallel reporter assay (lentiMPRA) to directly compare the functional activities of 2236 candidate liver enhancers in an episomal versus a chromosomally integrated context. We find that the activities of chromosomally integrated sequences are substantially different from the activities of the identical sequences assayed on episomes, and furthermore are correlated with different subsets of ENCODE annotations. The results of chromosomally based reporter assays are also more reproducible and more strongly predictable by both ENCODE annotations and sequence-based models. With a linear model that combines chromatin annotations and sequence information, we achieve a Pearson's $R^{2}$ of 0.362 for predicting the results of chromosomally integrated reporter assays. This level of prediction is better than with either chromatin annotations or sequence information alone and also outperforms predictive models of episomal assays. Our results have broad implications for how cis-regulatory elements are identified, prioritized and functionally validated.
\end{abstract}

[Supplemental material is available for this article.]

An enhancer is defined as a short region of DNA that can increase the expression of a gene, independent of its orientation and flexible with respect to its position relative to the transcriptional start site (Banerji et al. 1981; Moreau et al. 1981). Enhancers are thought to be modular, in the sense that they are active in heterologous sequence contexts and in that multiple enhancers may additively dictate the overall expression pattern of a gene (Shlyueva et al. 2014). They act through the binding of transcription factors, which recruit histone modifying factors, such as histone acetyltransferase (HAT) or histone methyltransferase (HMT). Enhancers are also associated with chromatin remodeling factors (e.g., SWI/ SNF) and the cohesin complex, which are involved in regulating chromatin structure and accessibility (Schmidt et al. 2010; Euskirchen et al. 2011; Faure et al. 2012).

Antibodies against specific transcription factors (TFs), histone modifications or transcriptional coactivators are commonly used

\footnotetext{
${ }^{7}$ These authors contributed equally to this work.

Corresponding authors: nadav.ahituv@ucsf.edu, shendure@uw.edu Article published online before print. Article, supplemental material, and publication date are at http://www.genome.org/cgi/doi/10.1101/gr.212092.116.
}

for chromatin immunoprecipitation followed by massively parallel sequencing (ChIP-seq) to identify candidate enhancers in a genome-wide manner. For example, the ENCODE Project Consortium and other efforts have identified thousands of candidate enhancers in mammalian genomes on the basis of such marks or their correlates (e.g., EP300 ChIP-seq, H3K27ac ChIP-seq, and DNase I hypersensitivity) in diverse cell lines and tissues (Visel et al.2009; The ENCODE Project Consortium 2012). However, a major limitation of such assays is that they reflect biochemical marks that are correlated with enhancer activity, rather than directly showing that any particular sequence actually functions as an enhancer. In other words, although such assays yield genome-wide catalogs of potential enhancers, they do not definitively predict bona fide enhancers nor precisely define their boundaries.

For decades, the primary means of functionally validating enhancers has been the episomal reporter assay. The standard

(C) 2017 Inoue et al. This article is distributed exclusively by Cold Spring Harbor Laboratory Press for the first six months after the full-issue publication date (see http://genome.cshlp.org/site/misc/terms.xhtml). After six months, it is available under a Creative Commons License (Attribution-NonCommercial 4.0 International), as described at http://creativecommons.org/licenses/by-nc/4.0/. 
approach is to relocate the candidate enhancer sequence to an episomal vector, adjacent to a minimal promoter driving expression of a reporter gene, e.g., luciferase or others. More recently, massively parallel reporter assays (MPRAs) have enabled the functional characterization of cis-regulatory elements, including enhancers, in a high-throughput manner. MPRAs use sequencingbased quantification of reporter barcodes to enable multiplexing of the reporter assay (Patwardhan et al. 2009). MPRAs have been used primarily in an episomal manner for the saturation mutagenesis of promoters and enhancers (Patwardhan et al. 2009, 2012; Kinney et al. 2010; Melnikov et al. 2012), for exploring the grammatical rules of promoters and enhancers (Smith et al. 2013; Sharon et al. 2014), and for testing thousands of enhancer candidates in different cells or tissues (Kwasnieski et al. 2012; Arnold et al. 2013, 2014; Kheradpour et al. 2013; Shlyueva et al. 2014; Savic et al. 2015; White 2015). Adeno-associated virus (AAV) MPRAs have also been developed, allowing these assays to be carried out in vivo and to perform reporter assays within target cells and tissues that are difficult to transduce, such as the brain (Shen et al. 2016), although these do not involve genomic integration.

Despite their widespread use to validate enhancers and other cis-regulatory elements, a longstanding concern about reporter assays is that they are almost always carried out via transient transfection of nonintegrating episomes. It is unknown whether transiently transfected sequences are chromatinized in a way that makes them appropriate models for endogenous gene expression from chromosomes (Smith and Hager 1997), but to the extent that this question has been explored, there are differences. For example, work from Archer, Hager, and colleagues, using the mouse mammary tumor virus (MMTV) promoter as a model, shows differences in histone $\mathrm{H} 1$ stoichiometry and nucleosome positioning resulting in an inability of episomal assays to reliably assay cooperative TF binding (Archer et al. 1992; Smith and Hager 1997; Hebbar and Archer 2007, 2008). In other work, the chromatin structure of transiently transfected nonreplicating plasmid DNA was observed to be differently fragmented than endogenous chromatin by micrococcal nuclease and, along with other data, supports a model in which atypical chromatin might be induced by association of episomes with nuclear structures (Jeong and Stein 1994). However, the extent to which these factors operate to confound the results of reporter assays performed for enhancer validation, whether categorical (i.e., is a particular sequence an enhancer?), qualitative (i.e., in what tissues is an element an enhancer?), or quantitative (i.e., what level of activation does a particular sequence confer?), has yet to be systematically investigated.

To address these questions, we developed lentiviral MPRA (lentiMPRA), a technology that uses lentivirus to integrate enhancer MPRA libraries into the genome. To overcome the substantial position-effect variegation observed by others in attempting to use lentiviral infection for MPRA (Murtha et al. 2014), we used a flanking antirepressor element (\#40) and a scaffold-attached region (SAR) (Klehr et al. 1991; Kwaks et al. 2003) on either side of our construct. In addition, we relied on as many as 100 independent reporter barcode sequences per assayed candidate enhancer sequence, integrated at diverse sites. The resulting system allows for high-throughput, highly reproducible, and quantitative measurement of the regulatory potential of candidate enhancers in a chromosomally integrated context. Furthermore, the cell-type range of lentivirus transduction is much broader than transfection, e.g., permitting MPRAs to be conducted in neurons, primary cells, or organoids.

\section{Results}

\section{Construction and validation of the lentiMPRA vector}

The potential for confounding of lentiviral assays by site-of-integration effects was demonstrated by a recent MPRA study that used lentiviral infection and found that $26 \%$ of positive controls did not show activated GFP expression, whereas other measures estimated a false positive rate of $22 \%$ (Murtha et al. 2014). We therefore constructed a lentiviral vector ( $\mathrm{pLS}-\mathrm{mP}$ ) that contains a minimal promoter $(\mathrm{mP})$ and the enhanced green fluorescent protein $(E G F P)$ gene flanked on one side by the antirepressor element $\# 40$ and the other by a SAR (Fig. 1A; Supplemental File 1; Klehr et al. 1991; Kwaks et al. 2003; Kissler et al. 2006). In experiments involving chromosomal integration of this enhancer reporter, we confirmed that EGFP is not expressed in the absence of an enhancer, although abundantly expressed under the control SV4O enhancer across a panel of cell lines representing diverse tissues-of-origin. These include K562 (lymphoblasts), H1-ESC (embryonic stem cells), HeLa-S3 (cervix), HepG2 (hepatocytes), T47D (epithelial), and Sk-n-sh retinoic acid treated (neuronal) cells (Supplemental Fig. S1). Furthermore, when SV 40 and the Ltv 1 liver enhancer (Patwardhan et al. 2012) are tested without the flanking antirepressor sequences, we observed much lower levels of EGFP expression in HepG2 cells (Fig. 1B). FACS analysis showed that the inclusion of antirepressors increased the proportion of the cells that strongly express GFP (Fig. 1C). This result was consistent with our expectation that the antirepressors facilitate robust enhancermediated expression from the integrated reporter.

\section{Design and construction of a library of candidate liver enhancers}

To evaluate lentiMPRA, we designed a liver enhancer library that comprises 2236 candidate sequences and 204 control sequences (Fig. 1D; Supplemental File 2), each 171 bp in length. All enhancer candidate sequences were chosen on the basis of having ENCODE HepG2 ChIP-seq peaks for EP3OO and H3K27ac, which are generally indicative of enhancer function (Heintzman et al. 2007; Visel et al. 2009). A subset of candidates ("type 1") were centered at ChIP-seq peaks for forkhead box A1 (FOXA1) or FOXA2, known liver pioneer transcription factors (Lupien et al. 2008) or hepatocyte nuclear factor 4 alpha (HNF4A), a nuclear receptor involved in lipid metabolism and gluconeogenesis (Watt et al. 2003), while also overlapping with ENCODE-derived ChIP-seq peaks for the cohesin complex (RAD21 and SMC3) or chromodomain helicase DNA binding protein 2 (CHD2), a chromatin remodeler that is part of the SWI/SNF complex. Other subsets of candidates were required to overlap only a liver transcription factor peak ("type 2 "), only a chromatin remodeler peak ("type 3 "), or neither ("type 4"). The 204 control sequences comprised 200 synthetically designed controls from a previous study (synthetic regulatory element sequences [SRESs]; 100 positive and 100 negative) (Smith et al. 2013) and an additional two positive (pos1 and pos2) and two negative endogenous controls (neg1 and neg2). We confirmed by standard luciferase reporter assay that pos 1 and pos 2 showed weak and strong enhancer activity, respectively, whereas neg1 and neg2 showed no activity (Supplemental Fig. S2).

Each of the 2440 enhancer candidates or controls was synthesized in cis with 100 unique reporter barcodes on a 244,000-feature microarray (Agilent OLS; 15 bp primer +171 bp enhancer candidate or control +14 bp spacer +15 bp barcode +15 bp primer $=$ 230 mers). The purpose of encoding a large number of barcodes per assayed sequence was to facilitate reproducible and quantitative 
A

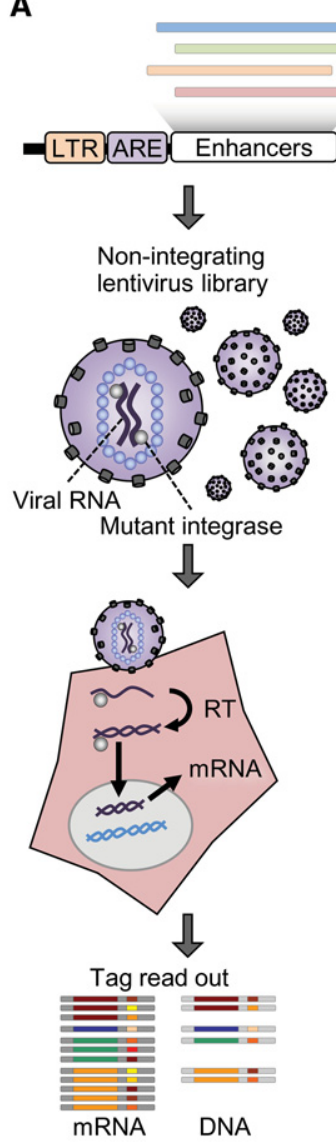

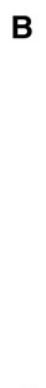

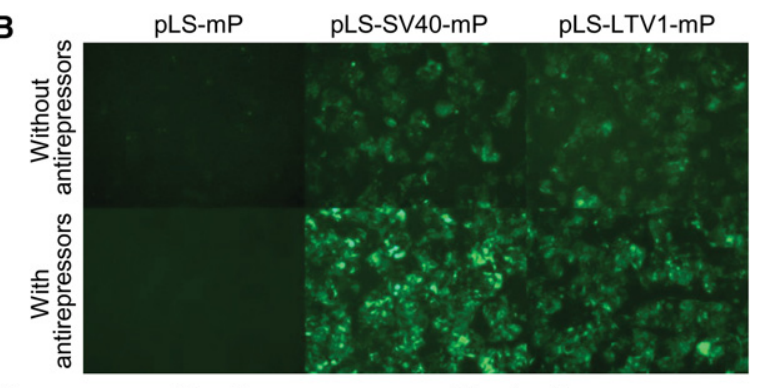

C

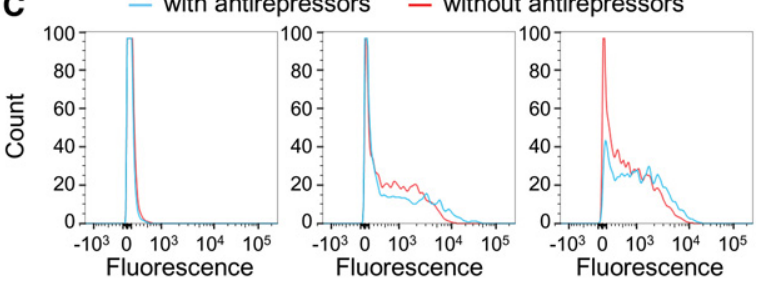

D

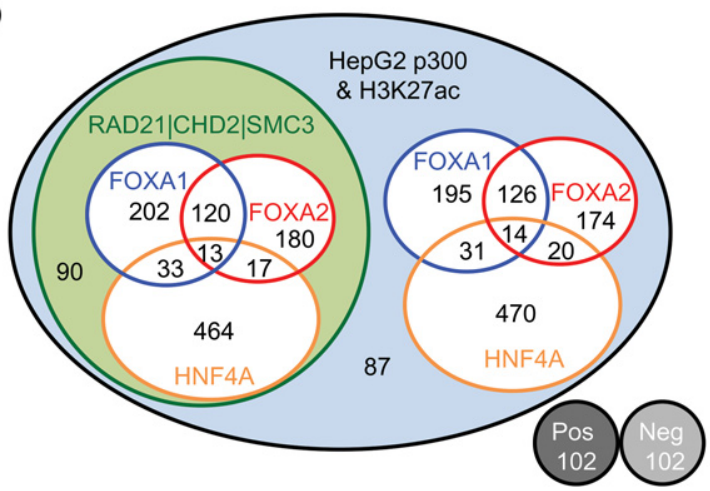

Figure 1. Study design for lentiMPRA. (A) Schematic diagram of lentiMPRA. Candidate enhancers and barcode tags were synthesized in tandem as a microarray-derived oligonucleotide library and cloned into the pLS-mP vector, followed by cloning of a minimal promoter (mP) and reporter (EGFP) between them. The resulting lentiMPRA library was packaged with either wild-type or mutant integrase and infected into HepG2 cells. Both DNA and mRNA were extracted, and the barcode tags were sequenced to test their enhancer activities in an episomal versus genome integrating manner. (B) HepG2 cells infected with lentiviral reporter construct bearing no enhancer (pLS-mP), an SV40 enhancer (pLS-SV40-mP), or Ltv1 (pLS-Ltv1-mP), a known liver enhancer (Patwardhan et al. 2012), with or without antirepressors. The inclusion of antirepressors results in stronger and more consistent expression, but is still dependent on the presence of an enhancer. (C) FACS analyses quantifying the fluorescence intensity of pLS-mP, pLS-SV40-mP, and pLS-Ltv1-mP with (blue lines) or without (red lines) antirepressors following infection into HepG2 with 1 copy of viral molecule per cell. Analysis of all GFP expressing cells (fluorescence more than 500 intensity units) shows a higher proportion of cells that strongly express GFP (more than 2000 units) when antirepressors are included. Specifically, $54.8 \%$ versus $45.1 \%$ for SV40, and $35.6 \%$ versus $29.0 \%$ for $L t v 1$, of cells with and without antirepressors, respectively. (D) Venn diagram showing the composition of the lentiMPRA library. Two thousand two hundred thirty-six enhancer candidate sequences were chosen on the basis of having ENCODE HepG2 ChIP-seq peaks for EP300 and H3K27ac marks. The candidates overlapped with or without ChIP-seq peaks for FOXA1, FOXA2, or HNF4A. Half the candidates overlapped with ChIP-seq peaks for RAD21, SMC3, and CHD2. In addition, the library included 102 positive and 102 negative controls.

measurements of regulatory activity, as well as to mitigate against nonuniformity in oligonucleotide synthesis. We cloned these oligonucleotides to a version of the lentiMPRA vector that lacked $\mathrm{mP}$ and EGFP reporter. Subsequently, a restriction site in the spacer was used to reinsert the $\mathrm{mP}+E G F P$ cassette between the candidate enhancer and barcode, thus positioning the barcode in the $3^{\prime}$ UTR of EGFP (Supplemental Fig. S3).

To evaluate the quality of the designed oligonucleotides and the representation of individual barcodes, we sequenced the cloned oligonucleotide library (i.e., prior to reinsertion of the $\mathrm{mP}+E G F P$ cassette) to a depth of 19.2 million paired-end consensus sequences, $52.6 \%$ of which had the expected length. Analysis of these data showed that most molecular copies of a given oligonucleotide are correct, that synthesis errors are distributed evenly along the designed insert sequence, and that single base deletions dominate the observed errors (Supplemental Fig. S4A). Nonetheless, there was substantial nonuniformity in the library (Supplemental Fig. S4B). Although $90.5 \%$ of the 244,000 designed barcodes were observed at least once among 11.0 million full-length barcodes sequenced, their abundance is sufficiently dispersed that we estimated that a subset of $56 \%-67 \%$ of the designed oligonucleotides would be propagated when maintaining a library complexity of 350,000-600,000 clones.

\section{Chromosomally integrated versus episomal lentiMPRA}

We next sought to directly compare the functional activities of the 2236 candidate liver enhancer sequences in a chromosomally integrated versus an episomal context. To this end, we packaged the lentiMPRA library with either a wild-type integrase (WT-IN) or a mutant integrase (MT-IN), with the latter allowing for the production of nonintegrating lentivirus and transient transgene

\section{Genome Research}

www.genome.org 
expression from nonintegrated DNA (Fig. 1A; Leavitt et al. 1996; Nightingale et al. 2006). Because the integrase is not encoded by the lentiMPRA library, this experimental design allows us to test the same exact library in both integrated and nonintegrated contexts.

To reduce background of unintegrated lentivirus in the integrating lentivirus prep, we obtained DNA/RNA from the cells with the WT-IN liver enhancer library at day 4 when they have an estimated 50 viral particles/cell and the MT-IN library at day 3 when they had an estimated 100 viral particles/cell (Supplemental Fig. S5A; for details, see Methods). The total copy number of viral DNA in the cells infected with the liver enhancer libraries was validated by qPCR (Supplemental Fig. S5B). During human immunodeficiency virus (HIV) infection, nonintegrating virus represents a major portion of the virus at early infection time points and includes linear DNA that is rapidly degraded along with circular DNA containing terminal repeats (1-LTRC and 2-LTRc) (Munir et al. 2013). We further confirmed the copy number of nonintegrated virus at our assayed time points by carrying out a qPCR on 2-LTRc, observing the expected low and high amounts of nonintegrated virus with $W T$-IN and MT-IN, respectively (Supplemental Fig. S5B).

\section{lentiMPRA on 2236 candidate liver enhancer sequences}

We recovered RNA and DNA from both WT-IN and MT-IN infections (three replicates each consisting of independent infections with the same library), amplified barcodes, and performed sequencing (Illumina NextSeq). We used both the forward and reverse reads to sequence the 15-bp reporter barcodes and obtain consensus sequences. We obtained an average of approximately 4.1 million raw barcode counts for DNA and an average of approximately 26 million raw barcode counts for RNA. Across replicates and sample types, $\sim 97 \%$ of barcodes were the correct length of $15 \mathrm{bp}$.

We matched the observed barcodes against the designed barcodes and normalized RNA and DNA for different sequencing depths in each sample by dividing counts by the sum of all observed counts and reporting them as counts per million. Only barcodes observed at least once in both RNA and DNA of the same sample were considered. Subsequently, RNA/DNA ratios were calculated. The average Spearman's rho for DNA counts of the three integrase mutant (MT) experiments was 0.907, and for RNA counts of the MT experiments was 0.982. The average Spearman's rho values for the wild-type integrase (WT) experiments were 0.864 and 0.979 for DNA and RNA, respectively. These correlations were determined for barcodes observed in pairs of replicates. Scatter plots for the MT and WT experiments are shown in Supplemental Figures S6 and S7, respectively.

Although the DNA and RNA counts for individual barcodes are highly correlated between experiments, the noise of each measure results in a poor correlation of RNA/DNA ratios (Supplemental Figs. S6, S7). However, there are on average 59-62 barcodes per candidate enhancer sequence (insert) in each replicate (out of 100 barcodes programmed on the array, with 40\% lost during cloning as discussed above) (Supplemental Fig. S8). To reduce noise, we summed up the RNA or DNA counts across all associated barcodes for each insert observed in a given experiment and recalculated RNA/DNA ratios (Supplemental Fig. S9). After this step, pairwise correlations of DNA and RNA counts of replicates are very high (average Spearman's rho MT-RNA 0.996, MT-DNA 0.994, WT-RNA 0.997, and WT-DNA 0.991). Figure 2 shows scatter plots and correlation values for per-insert RNA/ DNA ratios for the MT and WT experiments. RNA/DNA ratios show markedly improved reproducibility after summing across barcodes, with an average Spearman's rho of 0.908 (MT) and 0.944 (WT). In all pairwise comparisons of replicates, the integrated (WT) MPRA experiments exhibit a broader dynamic range and greater reproducibility than the episomal (MT) MPRA experiments. We also explored how stable the correlation of RNA/DNA ratios is between replicates by down-sampling the number of barcodes per insert or specifying an exact number of barcodes per insert (Supplemental Fig. S10). Again, the WT experiments show greater reproducibility, especially for inserts represented by fewer independent barcodes.

To combine replicates, we normalized the RNA/DNA ratios for inserts observed in each replicate by dividing by their median and then averaged this normalized RNA/DNA ratio for each insert across replicates (Figs. 2, 3A, red boxes). Figure 3A shows scatter plots of the resulting MT and WT RNA/DNA ratios colored by the type of insert and/or transcription factors considered in the design (Supplemental Fig. S11 shows RNA/DNA ratio ranges by type of insert). As noted above, we observe a broader dynamic range in the WT experiment. Furthermore, the Spearman correlation between MT and WT is 0.785 , which is considerably lower than the correlation observed when correlating replicates of the same experimental type (Spearman correlation of 0.908 [MT] and 0.944 [WT]). This is also the case in pairwise comparisons of MT versus WT replicates (i.e., prior to combining replicates) (Fig. 2, yellow boxes). Overall, these results show that there are substantial differences in regulatory activity between identical sequences assayed in an integrated versus episomal context.

Importantly, we can see clear separation of positive and negative controls. Figure 3, B and C, display RNA/DNA ratios obtained for the highest and lowest SRESs in the MT and WT experiments compared to their previously measured effects in HepG2. Although the highest and lowest SRESs are well separated in both experiments (Kolmogorov-Smirnov and Wilcoxon ranksum $P$-values below $2.2 \times 10^{-16}$ ), the WT experiment separates the highest and lowest SRE controls slightly better than the MT experiment (Kolmogorov-Smirnov test D 0.97 vs 0.95, Wilcoxon rank-sum test W 9951 vs 9937).

We next sought to assess whether any of our design categories (i.e., types 1-4 defined above, reflecting subsets of candidate enhancers with coincident liver TF and/or chromatin remodeler/ cohesin complex ChIP-seq peaks) might underlie the observed differences (Fig. 3A; Supplemental Fig. S11). We did not observe differences in expression range between type 1 and type 2 designs, which differ with respect to whether they overlap with ChIP-seq peaks for chromatin remodeling factors or members of the cohesin complex (Supplemental Table S1; Supplemental Fig. S16). Unexpectedly, type 3 and 4 designs, which unlike type 1 and 2 designs lack liver TF ChIP-seq peaks for FOXA1/2 and HNF4A, were more active. To attempt to explain overall differences in activity both for the different category types and in general, we next explored a broader set of genomic annotations and whether those are predictive of enhancer activity in the lentiMPRA assay.

\section{ENCODE and other genomic annotations that predict enhancer activity}

We evaluated whether genomic annotations, some numerical and other categorical (Supplemental File 3), were predictive of our results in HepG2 cells. The performance of individual numerical 


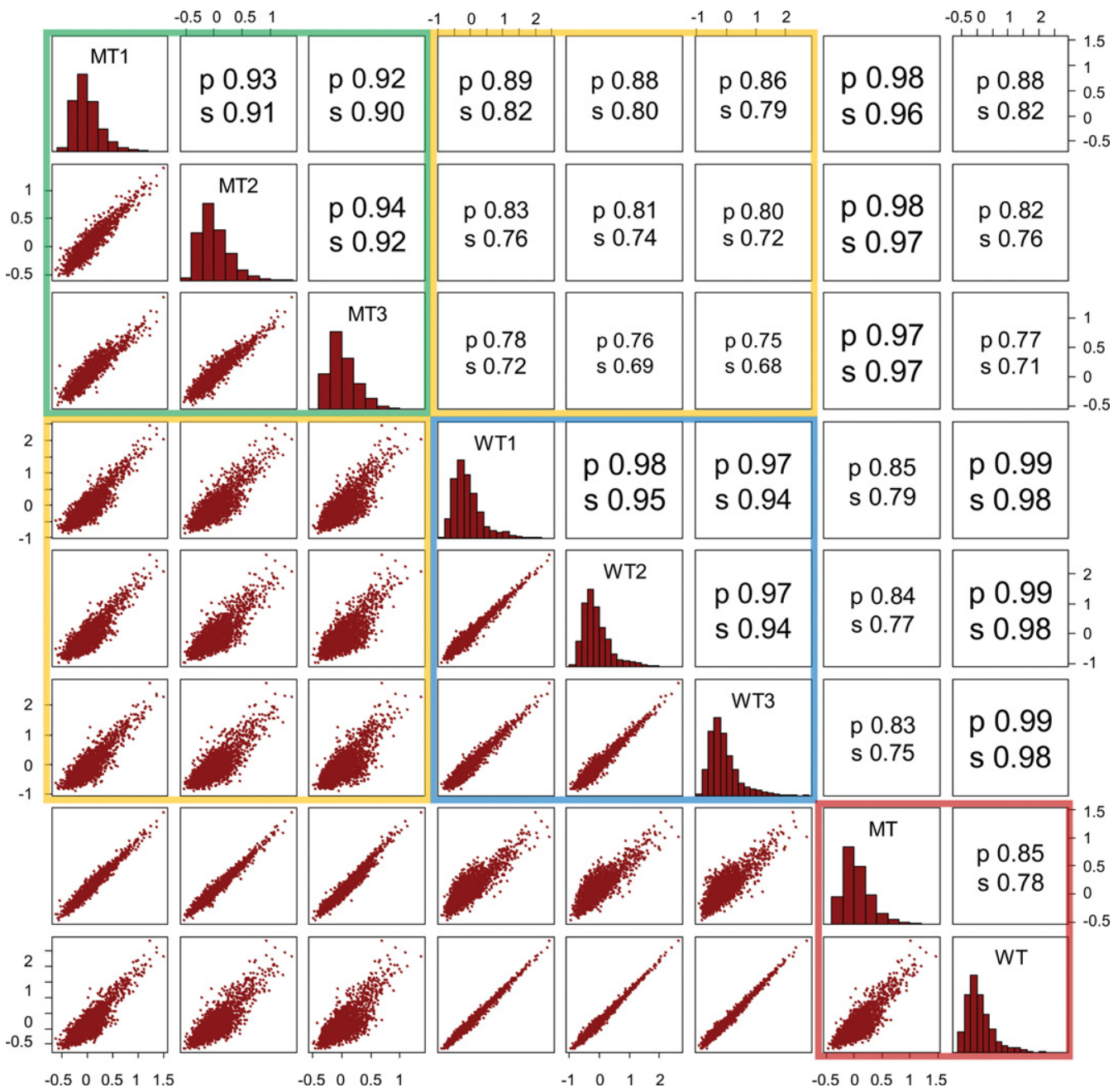

Figure 2. Pairwise correlation of per-insert RNA/DNA ratios between replicates, within and between MT versus WT experiments. The lower left triangle shows pairwise scatter plots. The diagonal provides replicate names and the respective histogram of the RNA/DNA ratios for that replicate. The upper triangle provides Pearson ( $p$ ) and Spearman (s) correlation coefficients. MT versus MT (green box) or WT versus WT (blue box) comparisons are substantially more correlated than MT versus WT (yellow boxes) comparisons, consistent with systematic differences between the episomal versus integrated contexts for reporter assays that exceed technical noise. The two right-most columns and two bottom-most rows correspond to MT and WT after combining across the three replicates, with the combined MT versus the combined WT comparison in the red box.

annotations for predicting the observed activity of candidate enhancer sequences are shown in Figure 4. We used Kendall's tau, a nonparametric rank correlation that is more conservative than Spearman's rho, because of the large number of zero values in our annotations that can result in artifacts from ties with Spearman's rho. In contrast with our design bins, many genomic annotations are observed to predict enhancer activity in both the WT and MT experiments. Across the board, annotations correlate better with the WT than the MT results, suggesting that integrated activity readouts (WT) correlate better with endogenous functional genomic signals (e.g., ChIP-seq data) than do episomal activity readouts (MT).

The most highly predictive numerical annotations, in both types of experiments, are HepG2 ChIP-seq data sets of JUND (Transcription Factor Jun-D) and FOSL2 (FOS-Like Antigen 2), consistent with a previous MPRA study that also highlighted the role of these transcription factors in HepG2 cells (Savic et al. 2015). For chromosomally based MPRA (WT), the number of overlapping ENCODE ChIP-seq peaks (TFBS) and the average ENCODE ChIP- seq signal (TFBSPeaks) as measured across different cell lines also rank among the more highly predictive annotations. However, these same features are the most discrepant with MT; that is, substantially less predictive of episomal MPRA. Of note, the highest observed $T^{2}$ for an individual annotation is only 0.033 (MT) and 0.059 (WT), highlighting the need for a model combining annotations and other available information (see below).

We also evaluated whether categorical annotations were predictive of our results (Supplemental Figs. S12-S15). Most of these annotations were derived for HepG2 cells by the ENCODE Project-ChromHMM (Ernst and Kellis 2012), SegWay (Hoffman et al. 2012), and Open Chromatin annotation. Although none of these were strongly predictive of the measured RNA/DNA ratios, the best performance was observed with the 25-state multiple cell-type SegWay or 15-state HepG2 ChromHMM chromatin segmentations. For SegWay, sequences annotated as TSS (transcription start sites) exhibited the highest expression, whereas sequences annotated as D (genomic death zones) exhibited the lowest expression.

\section{Genome Research}

www.genome.org 
A
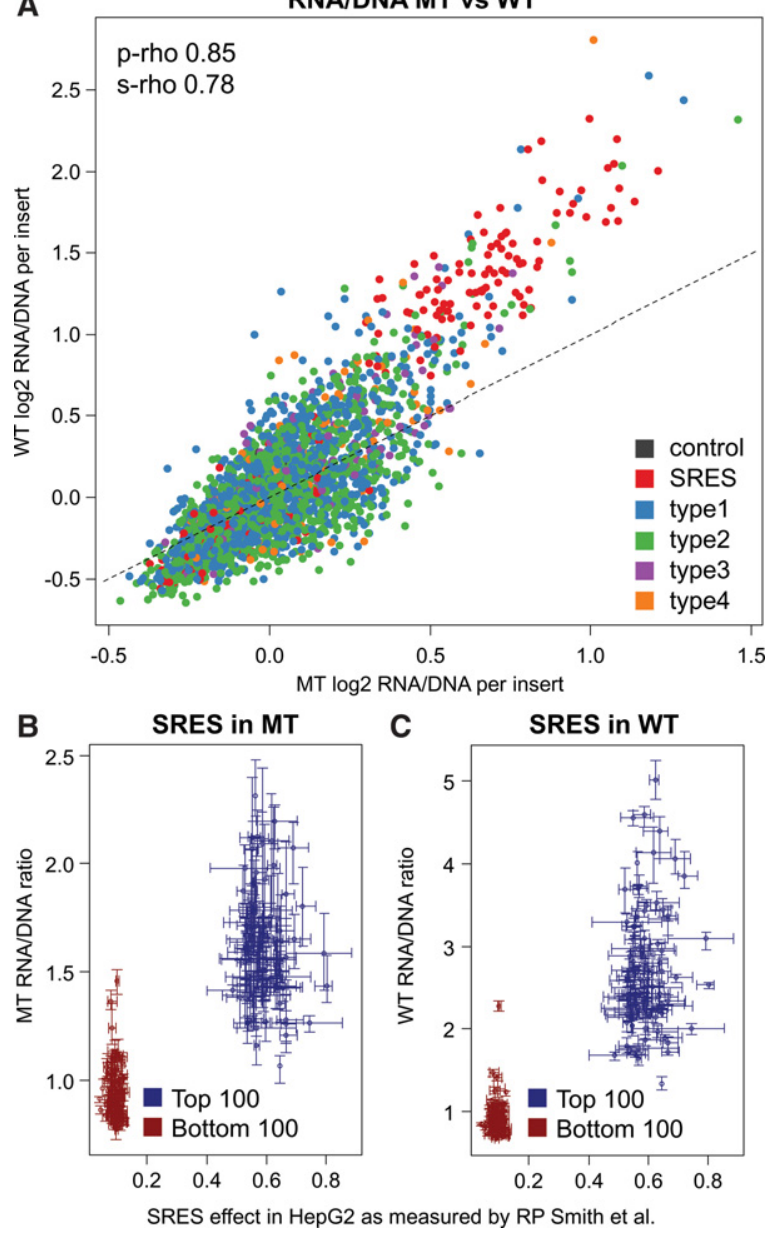

Figure 3. Comparisons between the nonintegrating (MT) and integrating (WT) libraries. (A) Scatter plot of combined MT versus WT RNA/DNA ratios. MT ratios show a smaller dynamic range and thus seem compressed compared to WT results. Data points are colored by the type of insert sequence, including two types of controls: a total of four positive and negative controls (black) as well as the highest 100 and lowest 100 synthetic regulatory element sequences (SRES, red) identified by Smith et al. (2013). The four classes of putative enhancer elements are the following: regions of FOXA1, FOXA2, or HNF4A binding that overlap H3K27ac and EP300 calls as well as at least one of three factors RAD21, CHD2, or SMC3 (type 1); regions like in type 1 but with no RAD21, CHD2, or SMC3 overlapping (type 2); EP300 peak regions overlapping H3K27ac as well as at least one overlap with RAD21,CHD2, or SMC3, but without peaks in FOXA1, FOXA2, or HNF4A (type 3); regions like in type 3 but with no RAD21, CHD2, or SMC3 overlapping (type 4). As shown here and in Supplemental Figure S11, we do not observe major differences between the four design types, either with respect to activity or MT versus WT. ( $B$, $C$ ) Enhancer activity of 200 synthetic regulatory element sequences (SRES) in the MT (B) and WT experiments (C). Scatter plot of RNA/DNA ratios for the top 100 positive and top 100 negative synthetic regulatory element (SRE) sequences in HepG2 experiments by Smith et al. (2013). Plots show the combined RNA/DNA ratios on the $y$-axis and measurements by Smith et al. (2013) on the $x$-axis. Intervals indicate the mean, minimum, and maximum values observed for three replicates performed with each experiment.

\section{Sequence-based predictors of functional activity}

Because sequences are removed from their native genomic context in the lentiMPRA assay, the relative ability of the elements that we test to increase transcription must be inherent to the 171-bp sequences themselves. It is therefore natural to ask whether it is pos- sible to build models for predicting functional activity that are based on primary sequence, rather than on genomic annotations. Although such models have historically been challenging to develop for mammalian genomes, Ghandi et al. (2014) recently introduced a "gapped $k$-mer" approach (gkm-SVM), with promising results for discriminating ENCODE ChIP-seq peaks versus matched control sequences. In brief, gkm-SVM trains a binary classifier based on the sets of $k$-mers that the training data contains (but allowing for some noninformative positions within $k$-mers, i.e., gaps). We collected all training data that Ghandi et al. (2014) used for HepG2, obtaining about 225,000 unique peak sequences as well as controls, and trained a combined, sequencebased model for predicting ChIP-seq peaks in HepG2 cells (Methods). Based on a set-aside test data set, the gkm-SVM model had a specificity of $71.8 \%$, a sensitivity of $88.8 \%$, and a precision of 75.9\% for separating ChIP-seq peak sequences from random control sequences.

We asked how well the resulting gkm-SVM scores correlated with the RNA/DNA ratios obtained for the MT and WT experiments (Fig. 5A,B). The combined gkm-SVM HepG2 model results in a Spearman's $R^{2}$ of 0.080 and 0.128 , for MT and WT, respectively. However, this correlation is at least partially driven by the synthetic control sequences, which can be scored with the sequencebased model but not with the genomic annotations. When excluding all control sequences, Spearman's $R^{2}$ values drop from 0.080 to 0.039 and from 0.128 to 0.076 for MT and WT, respectively. As such, there are a few ENCODE-based annotations which outperform the sequence-based gkm-SVM model, namely summaries of $J U N D / F O S L 2$ HepG2 ChIP-seq peaks, the number of overlapping ChIP-seq peaks (TFBS), or the average ChIP-seq signal (TFBSPeaks) measured across multiple ENCODE cell types.

\section{Combining annotations and sequence information to predict enhancer activity}

We next sought to combine information across multiple annotations to better predict enhancer activity. We fit Lasso linear models and selected the Lasso tuning parameter value by cross-validation (CV). Scatter plots as well as correlation coefficients were also obtained in a CV setup (Methods). We built models with all the genomic annotations described above (including the categorical annotations as binary features) as well as with and without the sequence-based gkm-SVM score from scaled and centered annotation matrixes (Supplemental Figs. S17-S19). SRESs and other controls were naturally excluded, as they are largely synthetic sequences and therefore missing genomic annotations. The resulting linear models were considerably more predictive of WT ratios than MT ratios (e.g., CV Spearman $R^{2}$ of 0.271 WT versus 0.148 MT; CV Pearson $R^{2}$ of 0.314 WT versus $0.194 \mathrm{MT}$ ). Including gkm-SVM scores in the models improved performance further (CV Spearman $R^{2}$ of 0.302 WT versus $0.156 \mathrm{MT}$; CV Pearson $R^{2}$ of 0.341 WT versus $0.203 \mathrm{MT}$ ). We noticed that gkm-SVM scores were assigned the largest model coefficients in both WT and MT models when they were included (Supplemental Fig. S19). Thus, although reasonably performing models are obtained from genomic annotations, the sequence-based gkm-SVM scores appear to capture independently predictive information.

We therefore decided to further explore sequence-based models with an improved implementation of gkm-SVM ("LS-GKM") (Lee 2016). We trained models from each of the 64 narrow-peak ChIP-seq data sets for which we had included summary statistics for the annotation matrix above (Methods). We then asked how 

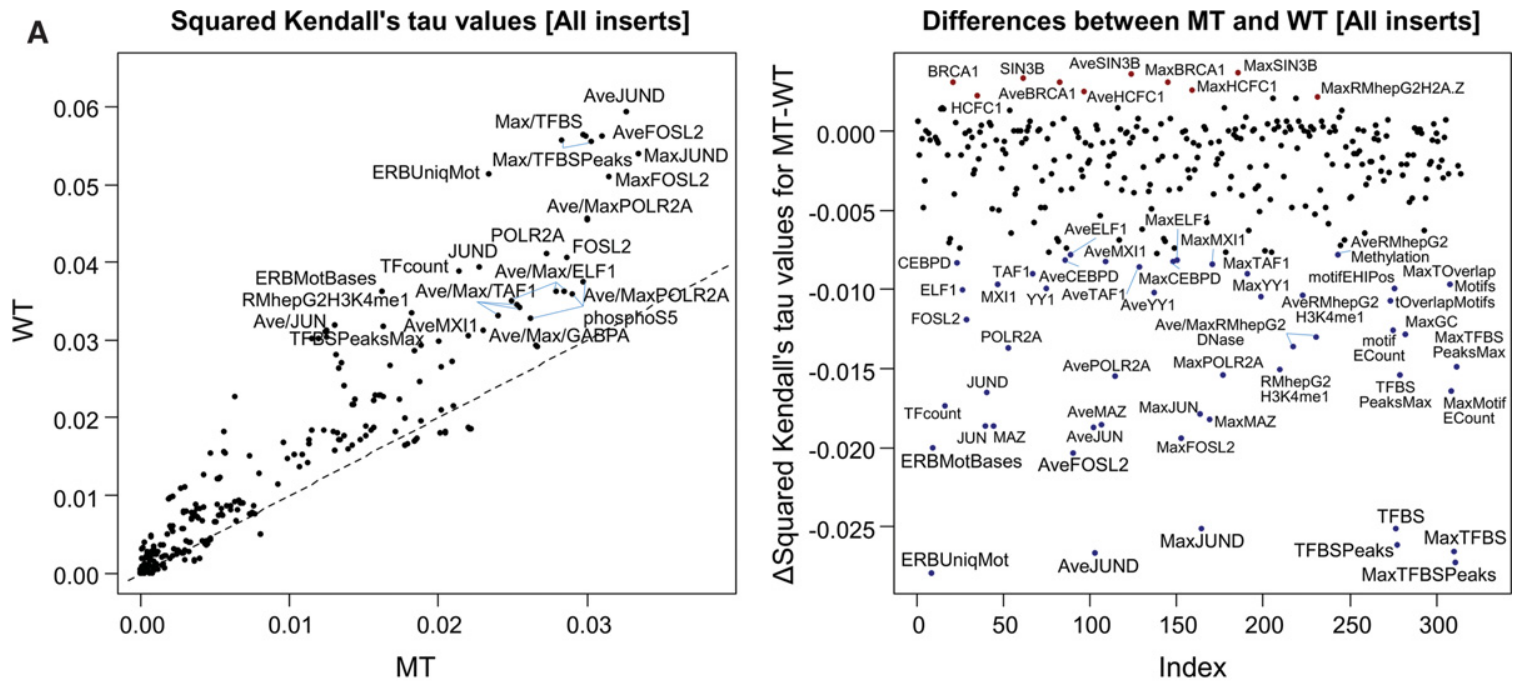

B Squared Kendall's tau values [Top 20\%]
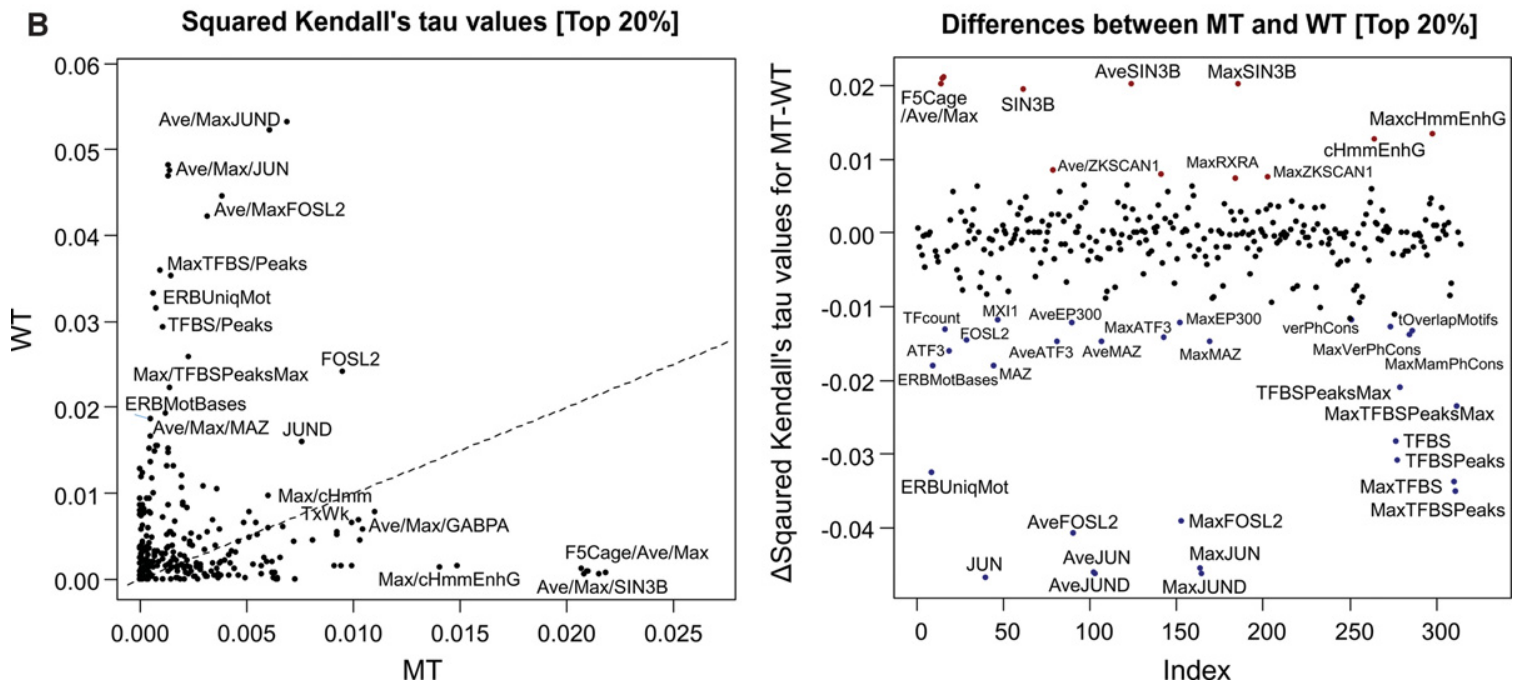

Figure 4. Squared Kendall's tau $\left(T^{2}\right)$ values for available genome annotations for predicting the activity of candidate enhancer sequences in the nonintegrating (MT) and integrating (WT) experiments. ( $A$ ) WT RNA/DNA ratios correlate better with annotations than the respective MT values. The left panel highlights the top correlated annotations for WT and MT ratios. The right panel highlights annotations with the largest difference in $T^{2}$ values between the MT and WT experiments. (B) Same analysis for the $20 \%$ most active elements (Supplemental Table S1).

well the LS-GKM scores generated by each of these 64 models predicted the results of the lentiMPRA experiments. Although the scores now correspond to sequence-based models of ChIP-seq peaks rather than the ChIP-seq peaks themselves, we once again observed the highest Spearman $R^{2}$ values for the individual factors JUND (0.120 WT/0.050 MT) and FOSL2 (0.108 WT/0.049 MT), and these are also the factors that show the largest differences in predictive value for WT versus MT (Supplemental Fig. S20). As such, sequence-based models of binding by these two factors as well as other individual factors exceed the performance of the pooled gkm-SVM sequence model.

We next fit Lasso linear models from the TF-specific LS-GKM SVM scores in order to predict the measured activities. The combined MT model (using 37 individual scores) achieves a CV Spearman $R^{2}$ of 0.128 (CV Pearson $R^{2}$ of 0.162 ), and the combined WT model (using 34 individual scores) of 0.227 (CV Pearson $R^{2}$ of 0.261) (Supplemental Fig. S21). This still falls short of models obtained purely from genomic annotations as described above. To test whether multiple ChIP-seq data sets should be combined in a sequence model rather than combining individual model scores in a linear model to improve prediction, we also trained LS-GKM models based on the peak sequences of the 37 (MT) and 34 (WT) scores selected by Lasso models as well as the top 2, 3, 5, 10, and top 15 coefficients in the Lasso models for MT and WT. We included coefficients independent of their direction (sign), but also trained only with sequences corresponding to a positive coefficient. Model performance increased when combining small numbers of peak sets and when limiting it to positive coefficients (Supplemental Table S2). Combining all or too many peaks in one sequence model reduced overall performance. From the combinations of ChIP-seq data sets tested here, the best performing sequence-based models achieved Spearman $R^{2}$ values of 0.054 (MT) and 0.133 (WT).

Finally, when we used both genomic annotations and the individual LS-GKM scores in a single linear model to predict the measured activities, performance increased to a CV Spearman $R^{2}$ of 0.168 (MT; Pearson $R^{2}$ of 0.206) and 0.322 (WT; CV Pearson $R^{2}$ of 0.362 ). These are our highest performing models predicting the

\section{Genome Research}

www.genome.org 
A MT RNA/DNA ratio vs HepG2 gkmSVM

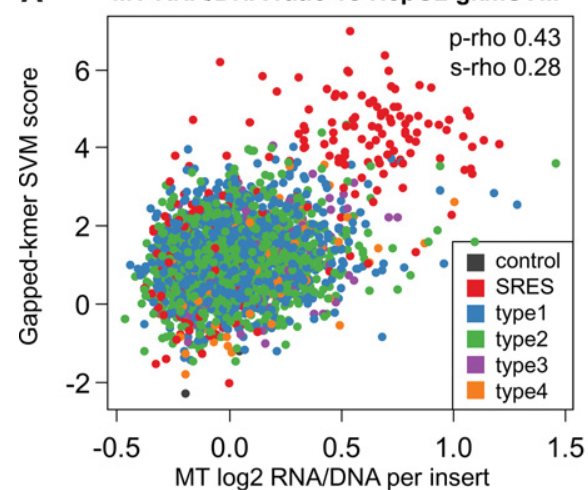

C

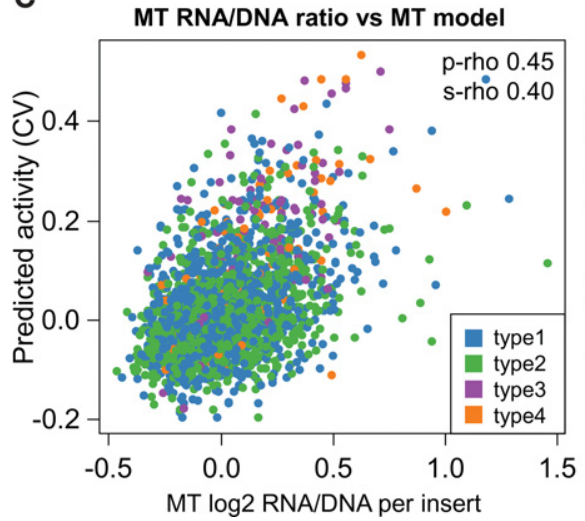

B WT RNA/DNA ratio vs HepG2 gkmSVm

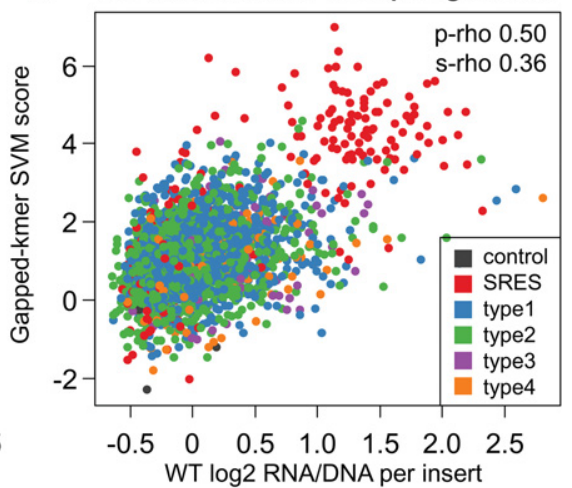

D

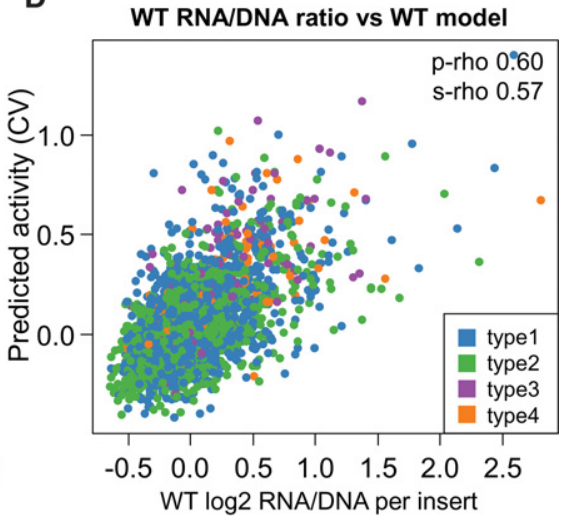

Figure 5. Prediction models. $(A, B)$ Correlation of gkm-SVM scores obtained for a combined HepG2 model with RNA/DNA ratios obtained from the mutant (MT) and wild-type integrase (WT) experiments. Data points are colored by the type of insert sequence, including two types of controls: 200 synthetic regulatory element sequences (SRES, red) identified by Smith et al. (2013), and four other control sequences (dark gray). The four classes of putative enhancer elements are the following: (type 1) regions of FOXA1, FOXA2, or HNF4A binding that overlap H3K27ac and EP300 calls as well as at least one of three factors RAD21, CHD2, or SMC3; (type 2) regions like in type 1 but with RAD21, CHD2, or SMC3; (type 3) EP300 peak regions overlapping H3K27ac as well as at least one overlap with RAD21, CHD2, or SMC3, but without peaks in FOXA1, FOXA2, or HNF4A; (type 4) regions like in type 3 but with no remodeling factor overlapping. Correlations are partially driven by the SRES; when excluding all controls, Spearman's $R^{2}$ values drop from 0.080 to 0.039 and from 0.128 to 0.076 for MT and WT, respectively. (C,D) Scatter plots of measured RNA/DNA ratios with predicted activity from linear Lasso models using annotations (numerical and categorical) as well as sequence-based (individual LS-GKM scores) information. Correlation coefficients are 0.45 Pearson/0.40 Spearman for the nonintegrated experiment (MT) and 0.60 Pearson/0.57 Spearman for the integrated constructs (WT). The models selected 110 (MT) and 133 (WT) of a total of 384 annotation features. Based on Pearson $R^{2}$ values, these combined models explain $20.6 \%$ (MT) and $36.2 \%$ (WT) of the variance observed in these experiments.

activities of candidate enhancer sequences for both the episomally and chromosomally encoded MPRA experiments (Fig. 5C,D).

\section{Discussion}

In this work, we report the first systematic comparison of episomal and chromosomally integrated reporter assays. Key aspects of our approach include (1) lentivirus-based MPRA or lentiMPRA, which can be used to in an episomal or integrated context by toggling whether a mutant versus wild-type integrase is used, and can furthermore be used in a wide variety of cell types, including neurons; (2) the use of numerous barcodes per candidate enhancer sequence, which results in highly reproducible measurements of transcriptional activation; and (3) extensive predictive modeling of our results, with the implicit assumption that a reasonable measure of a reporter assay's biological relevance is the extent to which it is correlated with endogenous genomic annotations.
We find that the results of integrated reporter assays are more reproducible, robust, and biologically relevant than episomal reporter assays. These conclusions are supported by the following observations: (1) We observed consistently greater reproducibility and dynamic range for the WT replicates as compared with the MT replicates; (2) the correlation of WT versus MT replicates (Spearman correlation of 0.785) was substantially lower than for WT versus WT (0.944) or MT versus MT (0.908), with clear systematic differences between the integrated and episomal contexts that exceed technical noise (Fig. 2; Supplemental Fig. S9); (3) the WT experiments were consistently more correlated with and more predictable by genomic annotations, which are based on biochemical marks measured in these sequences' native genomic contexts; and (4) many genomic annotations significantly predict the results of the WT but not the MT experiments.

Of note, we observed generally higher levels of expression with integrated reporters (Fig. 3A; Supplemental Table S1), consistent with previous findings that showed higher reporter gene levels for integrating relative to nonintegrating HIV-1 (Gelderblom et al. 2008; Thierry et al. 2016). However, it is worth noting that we used a lentivirus (not HIV-1) with a self-inactivating (SIN) LTR, which lacks viral promoters or enhancers, potentially influencing these expression differences. We also observed a larger variability between replicates for the MT condition. This variability could be explained by differences in histone structure, our assay being geared more for the WT condition (i.e., high MOI, many barcodes per sequence, anitrepressors), different time points ( $3 \mathrm{~d}$ for MT and $4 \mathrm{~d}$ for WT), suppression of transient plasmids (Qiu et al. 2011), and other factors. Results from hydrodynamic tail vein assays (which delivers reporter constructs into the mouse liver) also show that when chromatinized, plasmid DNA leads to higher expression levels than naked plasmid DNA (Kamiya et al. 2013). For HIV-1, both integrating and nonintegrating HIV-1 viral DNA are associated with histones (Kantor et al. 2009), which is probably also the case for our lentiviral vector. However, even if the lentiviral episome is chromatinized, there remain myriad potential causes for the observed differences in expression, including differences in H1 stoichiometry, nucleosome positioning, cooperative TF binding (Hebbar and Archer 2007, 2008), and/or nuclear location (Jeong and Stein 1994).

The number of overlapping ENCODE ChIP-seq peaks was one of the most strongly predictive annotations for our integrated sequences (Fig. 4, left). Interestingly, in experiments previously performed on the MMTV promoter, it was observed that nonintegrating constructs could not adequately assess cooperative TF 
binding due to differences in H1 stoichiometry and nucleosome positioning (Hebbar and Archer 2007), which may relate to the fact that this multiple TF binding was also one of the most differentiating annotations between the WT versus MT experiments (Fig. 4, right). Specific TF ChIP-seq-based sequence models that are similarly differentiating (Supplemental Fig. S19) include JUND, FOSL2, ATF3, and ELF1, which are known to interact and form complexes. Jun and Fos family members form the heterodimeric protein complex AP-1, which regulates gene expression in response to various stimuli including stress (Hess et al. 2004), and in the liver has known roles in hepatogenesis (Hilberg et al. 1993) and hepatocyte proliferation (Alcorn et al. 1990). AP-1 is known to form complexes with several additional protein partners (Hess et al. 2004), including ATF proteins such as ATF3 (Hai and Curran 1991) and ELF1, an ETS transcription factor (Bassuk and Leiden 1995). Of note, although lentivirus infection can induce stress potentially leading to increased expression of AP-1 and related factors, these same TFs were less predictive in MT-infected sequences; furthermore, the ChIP-seq data sets were generated on cells in normal physiological conditions. Combined, these findings suggest that differences in cooperative TF binding, possibly involving TFs including JUND, FOSL2, ELF1, and ATF3, might drive differences in the results of integrated versus episomal reporter assays. Interestingly, we observed a few histone related predictive annotations for the MT high expressing signals, including the SIN3 transcriptional regulator family member B (SIN3B), a scaffold protein that recruits chromatin modifying proteins (Kadamb et al. 2013) and histone variant $H 2 A . Z$, which has been shown to have important transcriptional activation roles (Subramanian et al. 2015). Although these annotations are based on the corresponding genomic DNA regions (e.g., ChIP-seq results) and only H1 stoichiometric differences were previously observed for the transient versus integrated MMTV promoter (Hebbar and Archer 2008), it would be interesting to test whether other histone differences exist for nonintegrating constructs on a larger scale.

Using single-feature models, we also systematically evaluated more than 400 genomic annotations and sequence models to explore which are significantly predictive of expression in the integrated and/or episomal lentiMPRA experiments (Supplemental Fig. S22). Consistent with our other analyses, there are many more annotations that are significantly predictive of the integrated (WT) assay but not the episomal (MT) assay. These include several annotations related to histone acetylation (HDAC2, EP300, and $Z B T B 7 A)$ as well as a factor $(C E B P B)$ with increased liver expression and which is associated with adipogenesis, gluconeogenic, and hematopoiesis (Tsukada et al. 2011). Interestingly, there are also a number of annotations that are only significantly predictive of the episomal assay, including BRCA1 and SIN $3 B$ binding motifs.

A limitation of this study and most contemporary MPRA experiments is that the length of the sequences tested are less than 200 bases. This may be addressable in the future through improvements to array-based oligonucleotide synthesis, multiplex DNA assembly protocols (Klein et al. 2016), or multiplex DNA capture protocols (Vockley et al. 2015). Furthermore, it remains uncertain what the true length distribution of enhancers is, although the size distribution of distal DNase I hypersensitive sites seems to provide a reasonable proxy of $300 \mathrm{bp}$ (Natarajan et al. 2012).

A contemporary challenge for our field is how to best identify, prioritize, and functionally validate cis-regulatory elements, especially enhancers. To address this, we envision a virtuous cycle, in which annotation and/or sequence-based models are used to nominate candidate enhancer sequences for validation, these candi- dates are tested in massively parallel reporter assays, and then the results are used to improve the models, which in turn results in higher quality nominations. Eventually, this will lead to not only a catalog of validated enhancers but also a deeper mechanistic understanding of the relationship between primary sequence, transcription factor binding, and quantitative enhancer activity. In this study, our best performing model achieves a Pearson's $R^{2}$ of 0.362 in predicting the results of the integrated lentiMPRA, with both genomic annotations and sequence-based models providing independent information. The result is encouraging, but the majority of variation remains unexplained. Of note, these are quantitative predictions of activity, a more challenging task than categorizing enhancers versus nonenhancers. Nonetheless, all the information underlying the differences is contained within the short sequences that we are testing and, in principle, should be learnable. Possible avenues for improving our predictive models include increasing the size of the regions tested so as to better align with the genomic annotations that we are using for prediction, additional annotations or alternative ways of summarizing available annotations, alternative sequence representations to gapped $k$ mers, allowing for interaction terms between model features, and markedly expanding the amount of training data. Indeed, with sufficient training data, we hope it will eventually be possible to accurately predict MPRA results from sequence-based models alone.

As our field scales MPRAs to characterize very large numbers of candidate enhancers, it is obviously critical that the reporter assays are as reflective as possible of endogenous biology. Our results directly test a longstanding concern about episomal reporter assays and suggest there are substantial differences between the integrated and episomal contexts. Furthermore, based on the fact that their output is more correlated with genomic annotations, we infer that integrated reporter assays are more reflective of endogenous enhancer activity. This fits with our expectation, as both the integrated reporter and endogenous enhancers reside within chromosomes as opposed to episomes.

Of course, an equally valid perspective on our observations is that reporter assays in the integrated versus episomal contexts are reasonably well correlated; therefore, the results of episomal assays remain informative of what activity would be in the integrated context (e.g., sequences observed to be strongly active in an episomal assay are likely to also be strongly active in an integrated assay). Whether the differences we observe here, with respect to activity, reproducibility, and correlation with genomic features, impact the interpretation of an episomal assay depends on the particulars of what was done and why (e.g., these differences may be more relevant for enhancer prediction and for quantitative modeling than for the binary classification of individual elements as activating versus inert). Furthermore, it is important to acknowledge that even integrated reporter assays are limited in important ways (e.g., by removing each tested element from its native sequence and epigenetic context). We therefore urge caution in the interpretation of the results of all reporter assays, but also that integrated reporter assays such as lentiMPRA be used where possible as an alternative to episomal reporter assays.

\section{Methods}

\section{Lentivirus enhancer construct generation}

To generate the lentivirus vector ( $\mathrm{pLS}-\mathrm{mP}$ ), a minimal promoter sequence, which originates from pGL4.23 (Promega), including an SbfI site was obtained by annealing of oligonucleotides (Sense:

\section{Genome Research}

www.genome.org 
5'-CTAGACCTGCAGGCACTAGAGGGTATATAATGGAAGCTCGA CTTCCAGCTTGGCAATCCGGTACTGTA-3'; Antisense: 5'-CCGG TACAGTACCGGATTGCCAAGCTGGAAGTCGAGCTTCCATTATA TACCCTCTAGTGCCTGCAGGT-3'; SbfI site is underlined) and

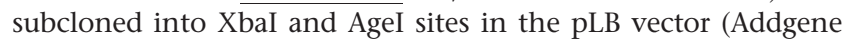
11619) (Kissler et al. 2006), replacing the U6 promoter and CMV enhancer/promoter sequence in the vector. To generate pLS-mP-SV40, the SV4O enhancer sequence was amplified from pGL4.13 (Promega) using primers (Forward: 5'-CAGGGCCCGC TCTAGAGCGCAGCACCATGGCCTGAA-3'; Reverse: 5'-TGCCTG CAGGTCTAGACAGCCATGGGGCGGAGAATG-3') and inserted into XbaI site in the vector using In-Fusion (Clontech). pos1, pos2, neg1, and neg2 sequences were amplified from human (pos1, neg2, pos2) or mouse (neg1) genome and inserted into EcoRV and HindIII site in pGL4.23 (Promega). Primers used are shown in Supplemental Table S3, and the annotated plasmid sequence file is available as Supplemental File 1.

\section{Library sequence design}

We picked 171-bp candidate enhancer sequences based on ChIPseq peaks calls for HepG2. We used narrow-peak calls for DNA binding proteins/transcription factors (FOXA1, FOXA2, HNF4A, RAD21, CHD2, SMC3, and EP300) and wide peak calls for histone marks (H3K27ac). We downloaded the call sets from the ENCODE portal (Sloan et al. 2016) (https://www.encodeproject.org/) with the following identifiers: ENCFFO01SWK, ENCFF002CKI, ENCFF002CKJ, ENCFF002CKK, ENCFF002CKN, ENCFF002CKY, ENCFF002CUS, ENCFF002CTX, ENCFF002CUU, ENCFF002CKV, and ENCFFO02CUN. We defined four types of sites: (1) regions centered over peak calls of $\leq 171$ bp for FOXA1, FOXA2, or $H N F 4 A$ that overlap H3K27ac and EP3OO calls as well as at least one of three factors: components of the cohesin complex (RAD21, SMC3) or chromatin remodeling factor CHD2; (2) regions like in type 1, but with no CHD2, RAD21, or SMC3 overlap; (3) regions of $171 \mathrm{bp}$ centered in an EP300 peak overlapping H3K27ac as well as at least one of three factors RAD21, CHD2, or SMC3, but without peaks in FOXA1, FOXA2, or HNF4A; and (4) regions like in type 3 but with no CHD2, RAD21, or SMC3 overlap. Sites of type 1 and 2 involving HNF4 were the most abundant sites and we used those to fill up our design after exhausting other target sequences.

Potential 171-bp target sequences were inserted into a 230-bp oligo backbone with a $5^{\prime}$ flanking sequence (15 bp, AGGACC GGATCAACT), 14-bp spacer sequence (CCTGCAGGGAATTC), 15-bp designed tag sequences (see below), and a 3' flanking sequence (15 bp, CATTGCGTGAACCGA) (Supplemental Fig. S3). Sequences were checked for SbfI and EcoRI restriction sites after joining the target sequence with the $5^{\prime}$ flanking sequence and the spacer sequence. Such potential target sequences were discarded.

In our final array design, we included 2440 different target sequences, each with 100 different barcodes (i.e., a total of 244,000 oligos). These included the highest 100 and lowest 100 synthetic regulatory element (SRE) sequences identified by Smith et al. (2013), four control sequences (neg1 MGSCv37 Chr 19: 35,531,983-35,532,154; neg2 GRCh37 Chr 5: 172,177,151172,177,323; pos1 GRCh37 Chr 3: 197,439,136-197,439,306; pos2 GRCh37 Chr 19: 35,531,984-35,532,154), which we tested using luciferase assays in the HepG2 cell line (Supplemental Fig. S2), 1029 type 1 inserts (202 FOXA1, 180 FOXA2, 464 HNF4A, 120 FOXA1\&FOXA2, 33 FOXA1\&HNF4A, 17 FOXA2\&HNF4A, 13 FOXA1\&FOXA2\&HNF4A), 1030 type 2 inserts (195 FOXA1, 174 FOXA2, 470 HNF4A, 126 FOXA1\&FOXA2, 31 FOXA1\&HNF4A, 20
FOXA2\&HNF4A, 14 FOXA1\&FOXA2\&HNF4A), 90 type 3 inserts, and 87 type 4 inserts.

Tag sequences of 15-bp length were designed to have at least two substitutions and one 1-bp insertion distance to each other. Homopolymers of length $3 \mathrm{bp}$ and longer were excluded in the design of these sequences, and so were ACA/CAC and GTG/TGT trinucleotides (bases excited with the same laser during Illumina sequencing). More than 556,000 such barcodes were designed using a greedy approach. The barcodes were then checked for the creation of SbfI and EcoRI restriction sites when adding the spacer and 3 ' flanking sequences. From the remaining sequences, a random subset of 244,000 barcodes was chosen for the design. The final designed oligo sequences are available in Supplemental File 2.

\section{Generation of MPRA libraries}

The lentiviral vector pLS-mP was cut with SbfI and EcoRI to temporarily liberate the minimal promoter and EGFP reporter gene. Array-synthesized 230-bp oligos (Agilent Technologies) containing an enhancer, spacer, and barcode (Supplemental Fig. S3) were amplified with adaptor primers (pLSmP-AG-f and pLSmPAG-r) that have overhangs complementary to the cut vector backbone (Supplemental Table S3), and the products were cloned using NEBuilder HiFi DNA Assembly mix (E2621). The adaptors were chosen to disrupt the original SbfI and EcoRI sites in the vector. The cloning reaction was transformed into electrocompetent cells (NEB C3020). Multiple transformations were pooled and midiprepped (Chargeswitch Pro Filter Plasmid Midi Kit, Invitrogen CS31104). This library of cloned enhancers and barcodes was then cut using SbfI and EcoRI sites contained within the spacer, and the minimal promoter and EGFP that were removed earlier were reintroduced via a sticky end ligation (T4 DNA Ligase, NEB M0202) between the enhancer and its barcode. These finished vectors were transformed and midi-prepped as previously mentioned.

\section{Quality control of designed array oligos}

Before inserting the minimal promoter and EGFP reporter gene, the plasmid library was sampled by high-throughput sequencing on an Illumina MiSeq $(206 / 200+6$ cycles) to check for the quality of the designed oligos and the representation of individual barcodes (sequencing primers are pLSmP-AG-seqR1, pLSmPAG-seqIndx, and pLSmP-AG-seqR2) (Supplemental Table S3). We sequenced the target, spacer, and tag sequences from both read ends and called a consensus sequence from the two reads. We obtained 19.2 million paired-end consensus sequences from this sequencing experiment, $52.6 \%$ of which had the expected length, $26.1 \%$ of sequences were 1 bp short, and $8.9 \%$ were 2 bp short (summing up to $87.6 \%$ ). Only $1.6 \%$ of sequences showed an insertion of $1 \mathrm{bp}$. These results are in line with expected dominance of small deletion errors in oligo synthesis. We aligned all consensus sequences back to all designed sequences using BWA MEM (Li and Durbin 2009) with parameters penalizing soft-clipping of alignment ends (-L 80). We consensus called reads aligning with the same outer alignment coordinates and SAM-format CIGAR string to reduce the effects of sequencing errors. We analyzed all those consensus sequences based on at least three sequences with a mapping quality above 0 . We note that substitutions are removed in the consensus calling process if the correct sequence is the most abundant sequence. Among these 992,513 consensus sequences, we observe instances of $91 \%$ designed oligos and $78 \%$ of oligos with one instance matching the designed oligo perfectly. Across all consensus sequences, the proportion of perfect oligos is only 19\%; however, the proportion vastly increases with the number of observations (69\% at 20 counts, $99 \%$ at 40 counts) 
(Supplemental Table S4). These observations are in agreement with most molecular copies of an oligo being correct, in combination with high representation differences in the library. Supplemental Figure S4A shows the distribution of alignment differences (as a proxy for synthesis errors) along the designed oligo sequences. Errors are distributed evenly along the designed insert sequence, with deletions dominating the observed differences. We observe that at some positions the deletion rate is reduced, whereas the insertion rate is increased. We speculate that this might be due to certain sequence contexts.

\section{Limited coverage of designed oligos in MPRA libraries}

From the analysis of oligo quality and oligo abundance above, we saw a first indication of the existence of a wide range of oligo abundance, and more frequent sequences tend to match the designed sequences perfectly (Supplemental Table S4). We characterized the abundance of oligos further and looked at the consequences that this has for generating libraries of lentivirus constructs with limited complexity (due to the transformation of a limited number of bacteria). Rather than looking at full-length oligos, we focused only on the tag sequences. Tag sequences were identified from the respective alignment positions of the alignments created above. To match the RNA/DNA count data analysis (see below), we only considered barcodes of 15-bp length (10.96 million/ $57.0 \%$, similar to the proportion of correct length sequences above). Of those 10.96 million barcodes, 345,247 different sequences are observed. We clustered (dnaclust) (Ghodsi et al. 2011) the remaining sequences allowing for one substitution and selecting the designed or most abundant sequence (reducing to 238,206 different sequences). The clustered sequences were matched against the designed barcodes (217,176 sequences, $99.2 \%$ of counts). The distribution of the abundance of these barcodes is available in Supplemental Figure S4B. We used those counts to simulate sampling from this overdispersed pool of sequences, as done when taking a sample of plasmids infusing the reporter gene and minimal promoter and again transforming the resulting plasmids. We sampled 10 times and averaged the number of unique designed barcodes: 150,000 clones - 87,944 unique barcodes, 250,000 clones - 116,297 unique barcodes, 350,000 clones - 135,222 unique barcodes, 500,000 clones - 154,090 unique barcodes, 600,000 clones $-163,831$ unique barcodes, 750,000 clones - 172,770 unique barcodes, and 1 million clones - 183,685 unique barcodes. Thus, even for high sampling depth, only a subset of barcodes will be captured in the final library. We observe on average 145,876 different barcodes which is concordant with more than 430,000 clones going into the lenti construction.

\section{Cell culture and GFP/luciferase assays}

HepG2 cells were cultured as previously described (Smith et al. 2013). K562, H1-ESC, HeLa-S3, T-47D, and Sk-n-sh cells were cultured as previously described (The ENCODE Project Consortium 2012). Sk-n-sh cells were treated with $24 \mu \mathrm{M}$ all trans-retinoic acid (Sigma) to induce neuronal differentiation. K562, H1-ESC, HeLa-S3, T-47D, and Sk-n-sh were infected with pLS-mP or pLSSV40-mP lentivirus along with $8 \mu \mathrm{g} / \mathrm{mL}$ polybrene and incubated for $2 \mathrm{~d}$, when they have an estimated 30, 60, 90, 90, and 90 viral particles/cell, respectively. The number of viral particles/cell was measured as described below. For the luciferase assay of the four control sequences (two negatives and two positives), we amplified the controls from the designed oligo pool (for primer sequences, see Supplemental Table S5) and inserted those into the pGL4.23 (Promega) reporter plasmid. $2 \times 10^{4} \mathrm{HepG} 2$ cells/well were seeded in a 96-well plate; $24 \mathrm{~h}$ later, the cells were transfected with $90 \mathrm{ng}$ of reporter plasmids (pGL4.23-neg1, pGL4.23-neg2, pGL4.23pos1, and pGL4.23-pos2) and 10 ng of pGL4.74 (Promega), which constitutively expresses Renilla luciferase, using X-tremeGENE HP (Roche) according to the manufacturer's protocol. The $\mathrm{X}$ tremeGENE:DNA ratio was 2:1. Three independent replicate cultures were transfected. Firefly and Renilla luciferase activities were measured as previously described (Smith et al. 2013).

\section{Lentivirus packaging, titration, and infection}

To optimize conditions and reduce background of unintegrated lentivirus in the integrating lentivirus prep, we utilized our positive control virus (pLS-SV40-mP) that was packaged with WT-IN and MT-IN, and we examined the viral titer by qPCR for three different volumes $(1,5$, and $25 \mu \mathrm{L}$ per well of a 24 -well plate) at four different time points ( $2-5 \mathrm{~d}$ post infection). For the lower volumes ( 1 and $5 \mu \mathrm{L}$ ), we observed a substantial reduction in total virus amounts at day 4 for both MT-IN and WT-IN that stabilized in the WT-IN only (Supplemental Fig. S5A). This suggests that the nonintegrated virus declines at this time point, similar to what was previously reported (Butler et al. 2001). For the high volume $(25 \mu \mathrm{L}$ ), we did not observe a substantial reduction or stabilization for MT-IN and WT-IN, respectively, until day 5 (Supplemental Fig. S5A), suggesting that high amounts of virus would make it difficult to distinguish between integrated and nonintegrated virus.

Twelve million HEK293T cells were plated in a $15-\mathrm{cm}$ dish and cultured for $24 \mathrm{~h}$. The cells were cotransfected with $8 \mu \mathrm{g}$ of the liver enhancer library and $4 \mu \mathrm{g}$ of packaging vectors using jetPRIME (Polyplus-transfections). psPAX2 that encodes wildtype pol was used to generate integrating lentivirus, whereas pLV-HELP (InvivoGen) that encodes a mutant pol was used to generate nonintegrating lentivirus. pMD2.G was used for both. The transfected cells were cultured for $3 \mathrm{~d}$, and lentiviruses were harvested and concentrated as previously described (Wang and McManus 2009).

To measure DNA titer for the integrating and nonintegrating lentivirus library, HepG2 cells were plated at $2 \times 10^{5}$ cells/well in 12 -well plates and incubated for $24 \mathrm{~h}$. Serial volume $(0,1,5$, $25 \mu \mathrm{L}$ ) of the lentivirus was added with $8 \mu \mathrm{g} / \mathrm{mL}$ polybrene, to increase infection efficiency. The infected cells were cultured for $2-5 \mathrm{~d}$ and then washed with PBS three times. Genomic DNA was extracted using the Wizard SV genomic DNA purification kit (Promega). Copy number of viral particle per cell was measured as the relative amount of viral DNA (WPRE region) over that of genomic DNA (intronic region of LIPC gene) by qPCR using SsoFast EvaGreen Supermix (Bio-Rad), according to manufacturer's protocol. PCR primer sequences are shown in Supplemental Table S3. For the lentiMPRA, 2.4 million HepG2 cells were plated on a 10$\mathrm{cm}$ dish and cultured for $24 \mathrm{~h}$. The cells were infected with integrating or nonintegrating lentivirus libraries along with $8 \mu \mathrm{g} / \mathrm{mL}$ polybrene and incubated for 4 and $3 \mathrm{~d}$, when they have an estimated 50 and 100 viral particles/cell, respectively. Three independent replicate cultures were infected. The cells were washed with PBS three times, and genomic DNA and total RNA was extracted using an AllPrep DNA/RNA mini kit (Qiagen). Copy number of viral particle per cell was confirmed by qPCR and shown in Supplemental Figure S5B. Messenger RNA (mRNA) was purified from the total RNA using Oligotex mRNA mini kit (Qiagen) and treated with Turbo DNase to remove contaminating DNA.

\section{RT-PCR, amplification, and sequencing of RNA/DNA}

For each replicate, $3 \times 500 \mathrm{ng}$ was reverse transcribed with SuperScript II (Invitrogen 18064-014) using a primer downstream from the barcode (pLSmP-ass-R-i\#) (Supplemental Table S3), 
which contained a sample index and a P7 Illumina adaptor sequence. The resulting cDNA was pooled and split into 24 reactions, amplified with Kapa Robust polymerase for three cycles using this same reverse primer paired with a forward primer complementary to the $3^{\prime}$ end of EGFP with a P5 adaptor sequence (BARCODE_ lentiF_v4.1) (Supplemental Table S3). The implemented tworound PCR setup is supposed to reduce PCR jack-potting effects and allows for incorporating unique molecular identifiers (UMIs), which could be used to correct for other PCR biases in future experiments. PCR products are then cleaned up with AMPure XP beads (Beckman Coulter) to remove the primers and concentrate the products. These products underwent a second round of amplification in eight reactions per replicate for 15 cycles, with a reverse primer containing only P7. All reactions were pooled at this point, run on an agarose gel for size-selection, and submitted for sequencing. For the DNA, $16 \times 500 \mathrm{ng}$ of each replicate was amplified for three cycles just as the RNA. First-round products were cleaned up with AMPure XP beads and amplified for another 16 reactions, each for 20 cycles. Reactions were pooled, gel purified, and sequenced. Sequencing primers are BARCODE-SEQ-R1-V4, pLSmP-AG-seqIndx, and BARCODE-SEQ-R2-V4 for both RNA and DNA barcodes (Supplemental Table S3).

RNA and DNA for each of three replicates were sequenced on an Illumina NextSeq instrument $(2 \times 26+10 \mathrm{bp}$ index $)$. The forward and reverse reads on this run each sequenced the designed 15-bp barcodes as well as an adjacent sequence to correctly trim and consensus call barcodes. We obtained a minimum of 2.9 million and a maximum of 5.9 million raw counts for DNA (average 4.1 million) and a minimum of 20.0 million and a maximum of 32.3 million raw counts for RNA (average 25.6 million). Across replicates and sample type, $97 \%$ of barcodes were of the correct length of $15 \mathrm{bp}$.

The number of unique sequences was on average 446,000 for DNA and 1.2 million for RNA. When clustering sequences with one substitution (dnaclust) (Ghodsi et al. 2011), the average number of unique sequences reduced to 280,000 for DNA and 697,000 for RNA. We speculate that our RNA readouts are impacted by sequence errors to a greater extent due to the reverse transcriptase (RT) step. When overlapping the observed with the designed sequences, clustering keeps more counts but reduces the total number of observed barcodes (93.1\% versus 90.3\%, 145,000 versus $151,000)$. We believe this is due to too many errors in barcodes that are sufficiently similar to cause clusters to merge across different designed tag sequences. We therefore dismissed the clustered data and only matched against the designed barcodes. This is further supported by counts being more highly correlated between replicates when using the nonclustered data (Spearman's rho without clustering: DNA replicates $88.6 \%$, RNA replicates $98.0 \%$; with clustering: DNA replicates $85.0 \%$, RNA replicates $94.3 \%$ ).

\section{Replicates, normalization, and RNA/DNA ratios}

To normalize RNA and DNA for different sequencing depths in each sample, we divided reads by the sum of observed counts and reported them as counts per million. Only barcodes observed in RNA and DNA of the same sample were considered. Subsequently, RNA/DNA ratios were calculated. We observe that the dynamic range observed in the WT experiments is larger and that the average Spearman's rho is also higher for the WT experiments (44.3\% versus 39.0\%). To determine the RNA/DNA ratios per insert, we summed up the counts of all barcodes contributing and determined the ratio of the average normalized counts. We explored how stable the correlation of RNA/DNA ratios is between replicates when limiting the number of barcodes per insert (Supplemental Fig S10). We limited the maximum number of bar- codes considered by (1) randomly down-sampling and (2) requiring an exact number of barcodes per insert (i.e., down-sampling those with more and excluding those inserts with less barcodes).

Although normalized individually, the three replicates of each experiment do not seem to be on the exact same scale (Fig. 2; Supplemental Fig. S9). We therefore chose to divide the RNA/ DNA ratios by the median across the technical replicate value before averaging them.

\section{Predictors of sequence effects}

To correlate available annotations with the observed sequence activity in HepG2 cells, we downloaded additional narrow-peak calls for DNA binding proteins/transcription factors in HepG2 from ENCODE data. We obtained call sets for the following 64 factors: ARID3A, ATF3, BHLHE40, BRCA1, CBX1, CEBPB, CEBPD, CHD2, CTCF, ELF1, EP3OO, EZH2, FOSL2, FOXA1, FOXA2, FOXK2, GABPA, GATA4, HCFC1, HDAC2, HNF4A, HNF4G, IRF3, JUN, JUND, MAFF, MAFK, MAX, MAZ, MBD4, MXI1, MYBL2, MYC, NFIC, NR2C2, NRF1, POLR2A, POLR2AphosphoS2, POLR2AphosphoS5, RAD21, RCOR1, REST, RFX5, RXRA, SIN3A, SIN3B, SMC3, SP1, SP2, SRF, TAF1, TBP, TCF12, TCF7L2, TEAD4, TFAP4, USF1, USF2, YY1, ZBTB33, ZBTB7A, ZHX2, ZKSCAN1, and ZNF274. Additionally, we downloaded ChromHMM segmentations for HepG2, Open Chromatin State, SegWay, and DHS call sets from the ENCODE portal (Sloan et al. 2016). From the NIH Roadmap Epigenomics Consortium, we obtained RNAseq, DNA methylation, DNase I, CAGE, H2A.Z, H3K4me1, H3K4me2, H3K4me3, H3K9ac, H3K9me3, H3K27ac, H3K27me3, H3K36me3, H3K79me2, H4K20me1, and ChromHMM segmentations tracks (Roadmap Epigenomics Consortium et al. 2015). We also downloaded the FANTOM5 Robust Enhancer annotations (Andersson et al. 2014), FANTOM5 CAGE data for HepG2 (FANTOM Consortium and the RIKEN PMI and CLST (DGT) et al. 2014), GenoSTAN Enhancer and promoter predictions (http://i12g-gagneurweb.in.tum.de/public/paper/GenoSTAN/), enhancerFinder predictions (Erwin et al. 2014), as well as motif scan results and annotated regulatory features from the Ensembl Regulatory Build (Zerbino et al. 2015). For further genome-wide and organismal metrics, we turned to the CADD v1.3 annotation file (Kircher et al. 2014) and extracted local GC and CpG content, SegWay, ChromHMM state across the NIH RoadMap cell types, priPhCons, mamPhCons, verPhCons, priPhyloP, mamPhyloP, verPhyloP, GerpN, GerpS, GerpRS, bStatistic, tOverlapMotifs, motifECount, motifEHIPos, TFBS, TFBSPeaks, TFBSPeaksMax, distance to TSS, and the actual CADD score column. We included the number of bases covered by peak calls as well as the average and maximum values across the designed sequences for those metrics. Supplemental File 3 outlines all annotations used.

\section{Gapped-k-mer SVM (gkm-SVM) model of HepG2 activity}

We collected training data of individual ChIP-seq binding factors described by Ghandi et al. (2014) for HepG2 (5000 specific ChIPseq peak regions and the same number of random controls; http:// www.beerlab.org/gkmsvm/) and removed duplicate sequences, obtaining 225,000 peak sequences as well as matched random controls. Attempting to train a classification model with the gkm-SVM software based on all peak sequences exceeded reasonable memory requirements (>1TB). Therefore, we iteratively reduced the number of training examples and ended up sampling each 50,000 peak and 50,000 control sequences for a combined HepG2 sequence model. Based on a test data set (2000 sampled from the unused training data set), the obtained model has a specificity of $71.8 \%$, a 
sensitivity of $88.8 \%$ and precision of $75.9 \%$ for separating ChIPseq peak from the random control sequences.

\section{Linear models integrating individual annotations}

We used the R glmnet package to fit Lasso-penalized linear models to predict RNA/DNA ratios. We used 10-fold cross-validation (cv. glmnet) to determine the Lasso tuning parameter lambda resulting in the minimum squared error. The Lasso forces small coefficients to zero, and thereby performs regression and feature selection simultaneously. Otherwise missing annotation values were mostly in count features $(70.1 \%)$ or absence of the conserved block annotation "GerpRS" (27.5\%), and thus all these values were imputed to zero. All annotation features were scaled and centered. Categorical features with $\mathrm{K}$ levels were included as K-1 binary columns. We excluded ZNF274 and EZH2 annotations from the model as none of the inserts overlapped with these ChIP-seq tracks. To report unbiased correlation values and scatter plots between the true and predicted RNA/DNA ratios, we randomly split up our data into 10 folds, trained models using nine folds and the above identified tuning parameter, and then extracted the fitted values after applying the model to the remaining fold.

\section{Sequence-based LS-GKM models}

LS-GKM (Lee 2016) is a faster and lower memory profile version of gkm-SVM. Its default settings are different from gkm-SVM (e.g., using 11 bases with seven informative positions rather than 10 bases with six informative positions). We applied LS-GKM using parameters corresponding to gkm-SVM (-l $10-\mathrm{k} 6$-d 3 -t 2 -T 4 -e 0.01$)$ as well as default parameters (-T 4 -e 0.01 ) on the HepG2 training data described for gkm-SVM above $(225,327$ positive/negative sequences each, 10,000 kept set aside for validation). We also compared performance for using the negative sequences as described for gkm-SVM (Ghandi et al. 2014) versus obtaining negative sequences by permutation of the real sequences maintaining dinucleotide content (Jiang et al. 2008). We found that best results were obtained for LS-GKM defaults in combination with selected negative sequences rather than permuted sequences (Supplemental Table S6). However, permuted sequences as a negative set produced a higher true positive rate and substantially simplify computation. We therefore used permuted sequence sets and ran LSGKM with default parameters for all models. We extracted genomic sequences (GRCh37) below the 64 ChIP-seq peak sets by concatenating multiple call sets for the same factor and merging overlapping peak regions using BEDTools (Quinlan and Hall 2010). We extracted up to $1 \mathrm{~kb}$ of sequence for each peak or centered 1-kb fragments on the peak for larger peak calls. We chose the model convergence parameter $e$ based on the number of positive training sequences (mean 16,600, min. 186, max. 63,948) multiplied with $1 \times 10^{-7}$; investing more training iterations for smaller training data sets.

We then used Lasso regression (as described above) to create combined models and we also trained LS-GKM models from pooled peak data sets (Supplemental Table S2). For this purpose, we pooled sequences using the peak data sets underlying the top $2,3,5,10$, and top 15 and all sequence models selected using Lasso regression.

\section{Individual feature models}

To explore whether certain annotations are more strongly predictive for either the nonintegrated (MT) or integrated (WT) expression measurements (despite the correlations among the annotations), we used the R glm (Generalized Linear Models) implementation to fit 430 linear single coefficient plus intercept models for predicting $\log _{2}$ RNA/DNA ratios for MT and WT experiments. We report the two-sided $P$-value for the t-statistic corresponding to the coefficient in the linear model, and used a significance criterion of 0.05 after Bonferroni correction (Supplemental Fig. S22; Supplemental Table S7).

\section{Data access}

The sequencing data, designed oligo sequences, and processed count and RNA/DNA ratio data including annotations have been submitted to the NCBI Gene Expression Omnibus (GEO; http://www.ncbi.nlm.nih.gov/geo/) under accession number GSE83894.

\section{Acknowledgments}

We thank members of the Ahituv, McManus, and Shendure laboratories for helpful discussions and suggestions. Our work was supported by the National Human Genome Research Institute (NHGRI) grant number 1R01HG006768 (N.A. and J.S.), NHGRI and Division of Cancer Prevention, National Cancer Institute grant number 1R01CA197139 (G.M.C., D.M.W., N.A., and J.S.), National Institute of Mental Health grant number 1R01MH109907 (N.A.), and the Uehara Memorial Foundation (F.I.). J.S. is an investigator of the Howard Hughes Medical Institute.

Author contributions: F.I., M.K., B.M., M.T.M., N.A., and J.S. designed experiments; F.I. and B.M. performed all wet laboratory experiments; M.K., J.S., N.A., D.M.W., and G.M.C. outlined data analysis; M.K. performed data analysis; F.I., M.K., N.A., and J.S. interpreted the experimental results; F.I., M.K., B.M., N.A., and J.S. wrote the manuscript.

\section{References}

Alcorn JA, Feitelberg SP, Brenner DA. 1990. Transient induction of c-jun during hepatic regeneration. Hepatology 11: 909-915.

Andersson R, Gebhard C, Miguel-Escalada I, Hoof I, Bornholdt J, Boyd M, Chen Y, Zhao X, Schmidl C, Suzuki T, et al. 2014. An atlas of active enhancers across human cell types and tissues. Nature 507: 455-461.

Archer TK, Lefebvre P, Wolford RG, Hager GL. 1992. Transcription factor loading on the MMTV promoter: a bimodal mechanism for promoter activation. Science 255: 1573-1576.

Arnold CD, Gerlach D, Stelzer C, Boryń ŁM, Rath M, Stark A. 2013. Genome-wide quantitative enhancer activity maps identified by STARR-seq. Science 339: 1074-1077.

Arnold CD, Gerlach D, Spies D, Matts JA, Sytnikova YA, Pagani M, Lau NC, Stark A. 2014. Quantitative genome-wide enhancer activity maps for five Drosophila species show functional enhancer conservation and turnover during cis-regulatory evolution. Nat Genet 46: $685-692$.

Banerji J, Rusconi S, Schaffner W. 1981. Expression of a $\beta$-globin gene is enhanced by remote SV40 DNA sequences. Cell 27(2 Pt 1): 299-308.

Bassuk AG, Leiden JM. 1995. A direct physical association between ETS and AP-1 transcription factors in normal human $\mathrm{T}$ cells. Immunity 3: 223-237.

Butler SL, Hansen MS, Bushman FD. 2001. A quantitative assay for HIV DNA integration in vivo. Nat Med 7: 631-634.

The ENCODE Project Consortium. 2012. An integrated encyclopedia of DNA elements in the human genome. Nature 489: 57-74.

Ernst J, Kellis M. 2012. ChromHMM: automating chromatin-state discovery and characterization. Nat Methods 9: 215-216.

Erwin GD, Oksenberg N, Truty RM, Kostka D, Murphy KK, Ahituv N, Pollard KS, Capra JA. 2014. Integrating diverse datasets improves developmental enhancer prediction. PLoS Comput Biol 10: e1003677.

Euskirchen GM, Auerbach RK, Davidov E, Gianoulis TA, Zhong G, Rozowsky J, Bhardwaj N, Gerstein MB, Snyder M. 2011. Diverse roles and interactions of the SWI/SNF chromatin remodeling complex revealed using global approaches. PLoS Genet 7: e1002008.

FANTOM Consortium and the RIKEN PMI and CLST (DGT), Forrest AR, Kawaji H, Rehli M, Baillie JK, de Hoon MJ, Haberle V, Lassmann T, 
Kulakovskiy IV, Lizio M, et al. 2014. A promoter-level mammalian expression atlas. Nature 507: 462-470.

Faure AJ, Schmidt D, Watt S, Schwalie PC, Wilson MD, Xu H, Ramsay RG, Odom DT, Flicek P. 2012. Cohesin regulates tissue-specific expression by stabilizing highly occupied cis-regulatory modules. Genome Res 22: 2163-2175.

Gelderblom HC, Vatakis DN, Burke SA, Lawrie SD, Bristol GC, Levy DN 2008. Viral complementation allows HIV-1 replication without integration. Retrovirology 5: 60.

Ghandi M, Lee D, Mohammad-Noori M, Beer MA. 2014. Enhanced regulatory sequence prediction using gapped $k$-mer features. PLoS Comput Biol 10: e1003711.

Ghodsi M, Liu B, Pop M. 2011. DNACLUST: accurate and efficient clustering of phylogenetic marker genes. BMC Bioinformatics 12: 271.

Hai T, Curran T. 1991. Cross-family dimerization of transcription factors Fos/Jun and ATF/CREB alters DNA binding specificity. Proc Natl Acad Sci 88: 3720-3724.

Hebbar PB, Archer TK. 2007. Chromatin-dependent cooperativity between site-specific transcription factors in vivo. J Biol Chem 282: 8284-8291.

Hebbar PB, Archer TK. 2008. Altered histone H1 stoichiometry and an absence of nucleosome positioning on transfected DNA. J Biol Chem 283: 4595-4601.

Heintzman ND, Stuart RK, Hon G, Fu Y, Ching CW, Hawkins RD, Barrera LO, Van Calcar S, Qu C, Ching KA, et al. 2007. Distinct and predictive chromatin signatures of transcriptional promoters and enhancers in the human genome. Nat Genet 39: 311-318.

Hess J, Angel P, Schorpp-Kistner M. 2004. AP-1 subunits: quarrel and harmony among siblings. J Cell Sci 117(Pt 25): 5965-5973.

Hilberg F, Aguzzi A, Howells N, Wagner EF. 1993. c-Jun is essential for normal mouse development and hepatogenesis. Nature 365: 179181.

Hoffman MM, Buske OJ, Wang J, Weng Z, Bilmes JA, Noble WS. 2012. Unsupervised pattern discovery in human chromatin structure through genomic segmentation. Nat Methods 9: 473-476.

Jeong S, Stein A. 1994. Micrococcal nuclease digestion of nuclei reveals extended nucleosome ladders having anomalous DNA lengths for chromatin assembled on non-replicating plasmids in transfected cells. Nucleic Acids Res 22: 370-375.

Jiang M, Anderson J, Gillespie J, Mayne M. 2008. uShuffle: a useful tool for shuffling biological sequences while preserving the k-let counts. BMC Bioinformatics 9: 192.

Kadamb R, Mittal S, Bansal N, Batra H, Saluja D. 2013. Sin3: insight into its transcription regulatory functions. Eur J Cell Biol 92: 237-246.

Kamiya H, Miyamoto S, Goto H, Kanda GN, Kobayashi M, Matsuoka I, Harashima H. 2013. Enhanced transgene expression from chromatinized plasmid DNA in mouse liver. Int J Pharm 441: 146-150.

Kantor B, Ma H, Webster-Cyriaque J, Monahan PE, Kafri T. 2009. Epigenetic activation of unintegrated HIV-1 genomes by gut-associated short chain fatty acids and its implications for HIV infection. Proc Natl Acad Sci 106: 18786-18791.

Kheradpour P, Ernst J, Melnikov A, Rogov P, Wang L, Zhang X, Alston J, Mikkelsen TS, Kellis M. 2013. Systematic dissection of regulatory motifs in 2000 predicted human enhancers using a massively parallel reporter assay. Genome Res 23: 800-811.

Kinney JB, Murugan A, Callan CG Jr, Cox EC. 2010. Using deep sequencing to characterize the biophysical mechanism of a transcriptional regulatory sequence. Proc Natl Acad Sci 107: 9158-9163.

Kircher M, Witten DM, Jain P, O'Roak BJ, Cooper GM, Shendure J. 2014. A general framework for estimating the relative pathogenicity of human genetic variants. Nat Genet 46: 310-315.

Kissler S, Stern P, Takahashi K, Hunter K, Peterson LB, Wicker LS. 2006. In vivo RNA interference demonstrates a role for Nramp1 in modifying susceptibility to type 1 diabetes. Nat Genet 38: 479-483.

Klehr D, Maass K, Bode J. 1991. Scaffold-attached regions from the human interferon $\beta$ domain can be used to enhance the stable expression of genes under the control of various promoters. Biochemistry 30: 1264-1270.

Klein JC, Lajoie MJ, Schwartz JJ, Strauch EM, Nelson J, Baker D, Shendure J. 2016. Multiplex pairwise assembly of array-derived DNA oligonucleotides. Nucleic Acids Res 44: e43.

Kwaks TH, Barnett P, Hemrika W, Siersma T, Sewalt RG, Satijn DP, Brons JF, van Blokland R, Kwakman P, Kruckeberg AL, et al. 2003. Identification of anti-repressor elements that confer high and stable protein production in mammalian cells. Nat Biotechnol 21: 553-558.

Kwasnieski JC, Mogno I, Myers CA, Corbo JC, Cohen BA. 2012. Complex effects of nucleotide variants in a mammalian cis-regulatory element. Proc Natl Acad Sci 109: 19498-19503.

Leavitt AD, Robles G, Alesandro N, Varmus HE. 1996. Human immunodeficiency virus type 1 integrase mutants retain in vitro integrase activity yet fail to integrate viral DNA efficiently during infection. J Virol 70: 721-728.

Lee D. 2016. LS-GKM: a new gkm-SVM for large-scale datasets. Bioinformatics 32: 2196-2198.

Li H, Durbin R. 2009. Fast and accurate short read alignment with BurrowsWheeler transform. Bioinformatics 25: 1754-1760.

Lupien M, Eeckhoute J, Meyer CA, Wang Q, Zhang Y, Li W, Carroll JS, Liu XS, Brown M. 2008. FoxA1 translates epigenetic signatures into enhancer-driven lineage-specific transcription. Cell 132: 958-970.

Melnikov A, Murugan A, Zhang X, Tesileanu T, Wang L, Rogov P, Feizi S, Gnirke A, Callan CG Jr, Kinney JB, et al. 2012. Systematic dissection and optimization of inducible enhancers in human cells using a massively parallel reporter assay. Nat Biotechnol 30: 271-277.

Moreau P, Hen R, Wasylyk B, Everett R, Gaub MP, Chambon P. 1981. The SV40 72 base repair repeat has a striking effect on gene expression both in SV40 and other chimeric recombinants. Nucleic Acids Res 9: 6047-6068.

Munir S, Thierry S, Subra F, Deprez E, Delelis O. 2013. Quantitative analysis of the time-course of viral DNA forms during the HIV-1 life cycle. Retrovirology 10: 87.

Murtha M, Tokcaer-Keskin Z, Tang Z, Strino F, Chen X, Wang Y, Xi X, Basilico C, Brown S, Bonneau R, et al. 2014. FIREWACh: high-throughput functional detection of transcriptional regulatory modules in mammalian cells. Nat Methods 11: 559-565.

Natarajan A, Yardımcı GG, Sheffield NC, Crawford GE, Ohler U. 2012. Predicting cell-type-specific gene expression from regions of open chromatin. Genome Res 22: 1711-1722.

Nightingale SJ, Hollis RP, Pepper KA, Petersen D, Yu XJ, Yang C, Bahner I, Kohn DB. 2006. Transient gene expression by nonintegrating lentiviral vectors. Mol Ther 13: 1121-1132.

Patwardhan RP, Lee C, Litvin O, Young DL, Pe'er D, Shendure J. 2009. Highresolution analysis of DNA regulatory elements by synthetic saturation mutagenesis. Nat Biotechnol 27: 1173-1175.

Patwardhan RP, Hiatt JB, Witten DM, Kim MJ, Smith RP, May D, Lee C, Andrie JM, Lee SI, Cooper GM, et al. 2012. Massively parallel functional dissection of mammalian enhancers in vivo. Nat Biotechnol 30: 265-270.

Qiu GH, Leung CH, Yun T, Xie X, Laban M, Hooi SC. 2011. Recognition and suppression of transfected plasmids by protein ZNF511-PRAP1, a potential molecular barrier to transgene expression. Mol Ther 19: 1478-1486.

Quinlan AR, Hall IM. 2010. BEDTools: a flexible suite of utilities for comparing genomic features. Bioinformatics 26: 841-842.

Roadmap Epigenomics Consortium, Kundaje A, Meuleman W, Ernst J, Bilenky M, Yen A, Heravi-Moussavi A, Kheradpour P, Zhang Z, Wang J, et al. 2015. Integrative analysis of 111 reference human epigenomes. Nature 518: 317-330.

Savic D, Roberts BS, Carleton JB, Partridge EC, White MA, Cohen BA, Cooper GM, Gertz J, Myers RM. 2015. Promoter-distal RNA polymerase II binding discriminates active from inactive CCAAT/enhancer-binding protein $\beta$ binding sites. Genome Res 25: 1791-1800.

Schmidt D, Wilson MD, Ballester B, Schwalie PC, Brown GD, Marshall A, Kutter C, Watt S, Martinez-Jimenez CP, Mackay S, et al. 2010. Five-vertebrate ChIP-seq reveals the evolutionary dynamics of transcription factor binding. Science 328: 1036-1040.

Sharon E, van Dijk D, Kalma Y, Keren L, Manor O, Yakhini Z, Segal E. 2014 Probing the effect of promoters on noise in gene expression using thousands of designed sequences. Genome Res 24: 1698-1706.

Shen SQ, Myers CA, Hughes AE, Byrne LC, Flannery JG, Corbo JC. 2016. Massively parallel cis-regulatory analysis in the mammalian central nervous system. Genome Res 26: 238-255.

Shlyueva D, Stampfel G, Stark A. 2014. Transcriptional enhancers: from properties to genome-wide predictions. Nat Rev Genet 15: $272-286$.

Sloan CA, Chan ET, Davidson JM, Malladi VS, Strattan JS, Hitz BC, Gabdank I, Narayanan AK, Ho M, Lee BT, et al. 2016. ENCODE data at the ENCODE portal. Nucleic Acids Res 44: D726-D732.

Smith CL, Hager GL. 1997. Transcriptional regulation of mammalian genes in vivo. A tale of two templates. J Biol Chem 272: 27493-27496.

Smith RP, Taher L, Patwardhan RP, Kim MJ, Inoue F, Shendure J, Ovcharenko I, Ahituv N. 2013. Massively parallel decoding of mammalian regulatory sequences supports a flexible organizational model. Nat Genet 45: 1021-1028.

Subramanian V, Fields PA, Boyer LA. 2015. H2A.Z: a molecular rheostat for transcriptional control. F1000Prime Rep 7: 01.

Thierry S, Thierry E, Subra F, Deprez E, Leh H, Bury-Moné S, Delelis O. 2016. Opposite transcriptional regulation of integrated vs unintegrated HIV genomes by the NF-кB pathway. Sci Rep 6: 25678.

Tsukada J, Yoshida Y, Kominato Y, Auron PE. 2011. The CCAAT/enhancer (C/EBP) family of basic-leucine zipper (bZIP) transcription factors is a 
Inoue et al.

multifaceted highly-regulated system for gene regulation. Cytokine 54: 6-19.

Visel A, Blow MJ, Li Z, Zhang T, Akiyama JA, Holt A, Plajzer-Frick I, Shoukry M, Wright C, Chen F, et al. 2009. ChIP-seq accurately predicts tissuespecific activity of enhancers. Nature 457: 854-858.

Vockley CM, Guo C, Majoros WH, Nodzenski M, Scholtens DM, Hayes MG, Lowe WL Jr, Reddy TE. 2015. Massively parallel quantification of the regulatory effects of noncoding genetic variation in a human cohort. Genome Res 25: 1206-1214.

Wang X, McManus M. 2009. Lentivirus production. J Vis Exp 32: 1499.
Watt AJ, Garrison WD, Duncan SA. 2003. HNF4: a central regulator of hepatocyte differentiation and function. Hepatology 37: 1249-1253.

White MA. 2015. Understanding how cis-regulatory function is encoded in DNA sequence using massively parallel reporter assays and designed sequences. Genomics 106: 165-170.

Zerbino DR, Wilder SP, Johnson N, Juettemann T, Flicek PR. 2015. The Ensembl Regulatory Build. Genome Biol 16: 56.

Received June 30, 2016; accepted in revised form November 8, 2016.

\section{Genome Research}




\title{
Corrigenda
}

Genome Research 28: 256-265 (2018)

\section{Corrigendum: Detecting differential copy number variation between groups of samples}

Craig B. Lowe, Nicelio Sanchez-Luege, Timothy R. Howes, Shannon D. Brady, Rhea R. Daugherty, Felicity C. Jones, Michael A. Bell, and David M. Kingsley

Due to a formatting error, Supplemental Table S4 in the above-mentioned article displayed incorrect gene identifiers as the closest transcription start site in column E. The revised Supplemental Table S4 has been posted online to reflect the correct gene identifiers. This error does not affect the analyses performed in this paper. The authors apologize for the inconvenience.

doi: $10.1101 /$ gr.237370.118

Genome Research 28: 145-158 (2018)

\author{
Corrigendum: Evolutionary expansion of DNA hypomethylation in the mammalian germline \\ genome \\ Jianghan Qu, Emily Hodges, Antoine Molaro, Pascal Gagneux, Matthew D. Dean, Gregory J. Hannon, \\ and Andrew D. Smith
}

The authors would like to correct the omission of a funding source in the Acknowledgments section which was inadvertently excluded from the initial publication of this article. The corrected text is as follows and has been updated online:

"This research was partially supported by National Institutes of Health grant R01 HG005238 to A.D.S. and National Institutes of Health grant R01 GM098536 to M.D.D."

The authors apologize for the inconvenience.

doi: $10.1101 /$ gr.236885.118

Genome Research 27: 38-52 (2017)

Corrigendum: A systematic comparison reveals substantial differences in chromosomal versus episomal encoding of enhancer activity

Fumitaka Inoue, Martin Kircher, Beth Martin, Gregory M. Cooper, Daniela M. Witten, Michael T. McManus, Nadav Ahituv, and Jay Shendure

The authors would like to correct the omission of Supplemental Files 1, 2, and 3 in the initial publication of this article. Please note that the Supplemental files have now been added to Supplemental Material online. The authors apologize for any confusion this may have caused. 


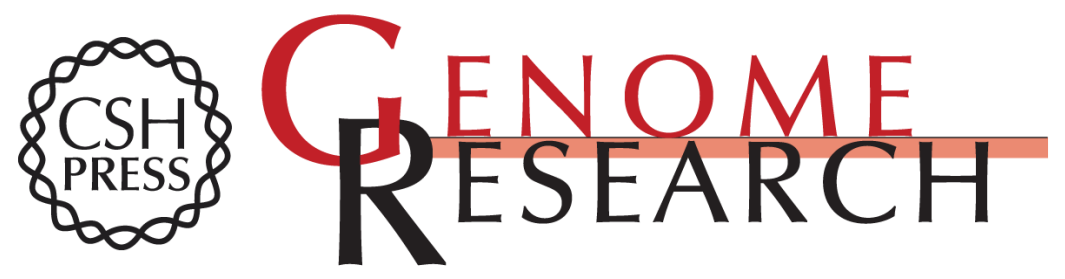

\section{A systematic comparison reveals substantial differences in chromosomal versus episomal encoding of enhancer activity}

Fumitaka Inoue, Martin Kircher, Beth Martin, et al.

Genome Res. 2017 27: 38-52 originally published online November 9, 2016

Access the most recent version at doi:10.1101/gr.212092.116

\section{Supplemental http://genome.cshlp.org/content/suppl/2016/12/19/gr.212092.116.DC1 \\ Material}

Related Content Corrigendum: A systematic comparison reveals substantial differences in chromosomal versus episomal encoding of enhancer activity Fumitaka Inoue, Martin Kircher, Beth Martin, et al. Genome Res. May, 2018 28: 766.3

References This article cites 69 articles, 19 of which can be accessed free at: http://genome.cshlp.org/content/27/1/38.full.html\#ref-list-1

Articles cited in:

http://genome.cshlp.org/content/27/1/38.full.html\#related-urls

Creative This article is distributed exclusively by Cold Spring Harbor Laboratory Press for the Commons first six months after the full-issue publication date (see

License

http://genome.cshlp.org/site/misc/terms.xhtml). After six months, it is available under a Creative Commons License (Attribution-NonCommercial 4.0 International), as described at http://creativecommons.org/licenses/by-nc/4.0/.

Email Alerting Receive free email alerts when new articles cite this article - sign up in the box at the Service top right corner of the article or click here.

\section{Affordable, Accurate Sequencing.}

To subscribe to Genome Research go to: https://genome.cshlp.org/subscriptions 